\title{
A Study on Force-based Collaboration in Swarms
}

\author{
Chiara Gabellieri $^{2}$. Marco Tognon ${ }^{1, \dagger}$. \\ Dario Sanalitro $^{1}$. Lucia Pallottino ${ }^{2}$. \\ Antonio Franchi ${ }^{1}$
}

\begin{abstract}
Cooperative manipulation is a basic skill in groups of humans, animals, and in many robotic applications. Besides being an interesting challenge, communication-less approaches have been applied to groups of robots in order to achieve higher scalability and simpler hardware and software design. We present a generic model and control law for robots cooperatively manipulating an object, for both ground and floating systems.

The control method exploits a leader-follower scheme and is based only on implicit communication (i.e., the sensing of contact forces). The control objective mainly consists of steering the object manipulated by the swarm of robots to a desired position and orientation in a cooperative way. For a system with just one leader, we present analytical results on the equilibrium configurations and their stability that are then validated by numerical simulations. The role of object internal forces (induced by the robots through contact forces) is discussed in terms of convergence of the object position and orientation to the desired values. We also present a discussion on additional properties of the controlled system that were investigated using thorough numerical analysis, namely, the robustness of the system when the object is subject to external disturbances in non-ideal conditions, and how the number of leaders in the swarm can affect the aforementioned convergence and robustness.
\end{abstract}

Keywords Cooperative manipulation · Communication-less coordination

This research was partially supported by the ANR, Project ANR-17-CE33-0007 MuRoPhen.

\author{
A. Franchi \\ E-mail: antonio.franchi@laas.fr \\ $\dagger$ The first two authors have equally collaborated to the manuscript and can both be considered \\ as first author. \\ ${ }^{1}$ LAAS-CNRS, Université de Toulouse, CNRS, Toulouse, France \\ ${ }^{2}$ Centro di Ricerca E. Piaggio, Dipartimento di Ingegneria dell'Informazione, Università di \\ Pisa, Italy
}




\section{Introduction}

Physical manipulation of the environment is one of the basic and most important ways in which humans, animals, and robots interact with the external world. Object manipulation is the basis for food retrieval for many biological systems, as well as for construction and deployment of items of any kind. If we think about robotic applications in our society, we find out that many of them are based on object manipulation: from logistics processes in warehouses to goods deployment in search and rescue missions. For these reasons, object manipulation is a significantly interesting problem to investigate, and the rich literature of the last decades witnesses it.

In this paper, we focus on the very attractive topic of cooperative manipulation of items by a group of robots. A single robot alone might not be strong enough to manipulate too heavy or too large objects. On the other hand, multiple robots can cooperatively carry out the manipulation task, overcoming, all together, the limitations of a single robot.

Several examples of cooperative manipulation in robotic (Yan et al., 2013) and even in mixed human-robot systems (Takubo et al. (2001), Ikeura et al. (1994)) have been reported in the literature. Centralized cooperative manipulation approaches, in which the knowledge of the entire system state is supposed to be available to at least one robot, are presented in Schneider and Cannon (1992) and A. Moosavian et al. (2005). Marino (2018) presents a control policy for cooperative ground manipulation in which the control law of each robot is based on the knowledge of the whole system state. The author also proposes a communication-based decentralized observer to estimate the quantities needed for the implementation of the control law.

Fully decentralized approaches have been the focus of several studies in the recent years because they grant the system with some desirable properties, such as scalability with respect to the number of robots, greater fault tolerance, and the need for a smaller amount of information that every single agent is required to know and to handle. Examples can be found for groups of ground (Petitti et al., 2016; Franchi et al., 2019), underwater (Conti et al., 2015) and aerial robots (Mellinger et al., 2013). The mentioned approaches are based on the explicit exchange of data among the robots. Other examples of decentralized approaches in which ground or aerial robots employ robotic manipulators can be found in Sieber and Hirche (2018) and Verginis et al. (2018).

Although many of the methods proposed in the literature are based on explicit communication within the group of robots, in this work we tackle the problem of cooperative manipulation without the use of explicit communication. Without data exchange between the robots, the communication-less approach allows simplifying the system architecture, both from the hardware and software points of view. Moreover, in cooperative manipulation systems, avoiding explicit communication allows overcoming typical communication problems among which power consumption, packets loss/corruption, and delays, which might lead to unstable behaviors of the system.

This work investigates the problem of cooperative object manipulation performed by groups of ground and floating robots. A control law based on a leaderfollower scheme is presented to steer the manipulated object to a desired configuration. The proposed approach does not rely on explicit communication but 
rather exploits only the measurements of the contact forces. Analytical results about the equilibria of the system and their stability are presented, highlighting the role of the internal forces in the object. Numerical simulations validate the results and show additional characteristics of the closed-loop system, such as its behavior under external disturbances and noisy measurements, and the effect of multiple leader robots.

The paper is structured as follows: Sec. 2 contains a description of the related literature and emphasizes the main differences with this work; in Sec. 3 the system dynamics is outlined; Sec. 4 defines the main hypothesis and the aim of the work; the theoretical results are drawn in Sec. 5, while the numerical results are reported in Sec. 6; finally, in Sec. 7 the results are discussed and summarized, and future developments are suggested.

\section{Related Work}

Besides being an interesting topic for real-world applications, objects manipulation by a swarm of robots solely based on implicit communication is also an inspiring challenge in itself. We can find different examples in nature that make us wonder whether we can reproduce the observed behavior in swarms of robots. Coordination mechanisms exploiting modifications of the external environment and requiring only implicit communication have been addressed as Stigmergy (Grassé, 1959). Of course, humans are capable of cooperatively manipulate objects without speaking, relying only on vision or force sensing. Communication-less cooperative manipulation has also been observed in nature (Berman et al., 2011; Kube and Bonabeau, 2000; Franks et al., 2001; Franks, 1986), and it has been regarded as the main coordination mechanism for items transportation in groups of ants (Kube and Bonabeau, 2000). A peculiar example of communication-less cooperative manipulation in biological systems is described by Di Leonardo et al. (2010), where unicellular self-propelling organisms, i.e., the bacteria Escherichia coli, put in an artificial environment, spontaneously arrange themselves around some artificial gears of the diameter of some tens of micrometers and make them spin. Many researchers, inspired by several examples from nature, studied how to replicate communication-less manipulation in multi-robot systems. While several works can be found in the literature concerning the swarms of ground robots (Kube and Bonabeau, 2000; Berman et al., 2011; Rubenstein et al., 2013; Wang and Schwager, 2016; Tuci et al., 2006; Farivarnejad and Berman, 2018), few of them consider aerial robots. In Kube and Bonabeau (2000), Berman et al. (2011), and Rubenstein et al. (2013) the effort has been put in replicating a specific behavior observed in groups of ants into a multi-robot setup. Instead, Wang and Schwager (2016) present a robotic framework where the attitude of the transported object is regulated by the leader, which can apply a pure torque to the object itself. The work by Farivarnejad and Berman (2018) is an example of communication-less object manipulation by point-mass robots rigidly attached to the object, which considers the planar problem of following a straight path at a desired velocity. Tsiamis et al. (2015) present one instance of object transportation by robotic manipulators equipped with a mobile base, rigidly attached to the object. Alternatively, in the last few years, UAVs (Unmanned Aerial Vehicles) have proven to be suitable for physical interaction using proper hardware design and control laws (Fumagalli 
et al., 2014; Tognon et al., 2019). Whereas different types of contact tools have been proposed (e.g., aerial robots equipped with robotic arms in Caccavale et al. (2015), spherical joints in Nguyen et al. (2015), fixed grippers in Mellinger et al. (2013)), cables have probably received most of the attention thanks to their light weight and mechanical simplicity (Gassner et al., 2017; Tagliabue et al., 2016; Tognon and Franchi, 2017; Sreenath and Kumar, 2013; Manubens et al., 2013; Pereira et al., 2016). Despite the advantages that aerial robotic swarms may present, such as a greater workspace, independence from uneven terrains, low cost and agility, only a few and very recent works can be found about communication-less transportation for these systems. Tagliabue et al. (2016) present experiments of two quadrotors transporting a cable-suspended beam-like load exploiting a leader-follower approach based on the force sensed by the follower robot; Gassner et al. (2017) show a similar experiment but based on vision; Tognon et al. (2018) also propose a thorough theoretical analysis for such a two-robot system.

The control architecture proposed in Tognon et al. (2018) is based on a leaderfollower scheme in which the robots, controlled by a compliant controller, are attached to a beam-like object by means of cables. The leader robot, aware of the desired direction of motion, steers the commonly manipulated object. On the other hand, the follower robot moves accordingly, by sensing the changes of force arisen in the cable due to the object motion. In this way, the manipulated object itself becomes the implicit-communication mean. Actually, it has also been observed in colonies of army ants like Eciton burchelli (Franks, 1986) and Dorylus wilverthi (Franks et al., 2001) that it is commonly a single ant, usually of a different cast, bigger and stronger than the others, that starts the motion during cooperative object transportation. The rest of the ants just follow. In more detail, in Tognon et al. (2018), the equilibria of the two-robots dynamic system are studied and their stability analyzed, highlighting the role of an internal force produced by the robots in the object. Internal forces are contact force distributions on an object that do not cause any motion. They compensate each other and only result in a stretch or compression of the object. Moreover, internal forces can be sensed by the robots and used for implicit communication.

Since the extension of analytic results to a swarm composed by an arbitrary number of robots, although very interesting, is not trivial (Abbasnejad and Carricato, 2015), in Gabellieri et al. (2018) a numerical study for this more general case is proposed. More specifically, Gabellieri et al. (2018) highlight the role of the internal forces in the convergence to a single configuration of equilibrium and in accelerating the convergence rate. Note that in the previous works about cooperative communication-based robotic manipulation where analytical proof of convergence is provided, such as Verginis et al. (2018) and Sieber and Hirche (2018), the robots are rigidly attached to the commonly transported object. As a consequence, the state of the object is known to each robot simply by the knowledge of their own end-effector position and orientation. The available information is explicitly exploited in the analytical derivation. Another instance of design and proof of convergence based on the rigid grasp of an object can be found in Tsiamis et al. (2015), where communication-less cooperative object manipulation in a 3D space has been presented. By contrast, in our framework, each robot cannot retrieve the object pose by the knowledge of its own state since the contact model allows an arbitrary relative orientation between the robot and the object, and the contacts are modeled by elastic elements. 
With this in mind, the aim of our work is to complete and further extend previous results in many directions. First of all, in this paper, we apply our control strategy to a more general family of systems, including also ground ones, hence addressing a wider class of possible applications and demonstrating the generality of the proposed control law.

If Gabellieri et al. (2018) consider only stretching internal forces, here we also show the effects of compressing internal forces to the convergence of the object to a desired configuration. Additionally, we report analytical results concerning the equilibrium configurations of the system with one leader, focusing on the stability/instability of such configurations. Through extensive numerical simulations, validating the derived theoretical results, we also show the behavior of the swarm under external disturbances. The results show the role of internal forces to enhance the robustness of the system against non-ideal conditions. We believe that this study is a crucial point for the applicability of the method to real systems characterized by uncertain and noisy conditions.

Eventually, we also propose a novel approach consisting of inserting more than one leader robot in the swarm, namely, more than one robot aware of the desired final configuration and direction of motion. The idea of having multiple leader robots in a swarm has been already proposed in other works such as in Ferrante et al. (2012) and in Ferrante et al. (2010). In Kelly and Keating (1996) the presence of multiple leader robots is exploited to allow the circumnavigation of large obstacles splitting the flock into separate but connected sub-groups, where each group needs at least one leader. Through an extensive set of numerical simulations, we study the stability and robustness of the system against non-ideal conditions when we vary the number of leaders. The results show that a swarm with no leader robots is capable of orienting the object to a desired attitude if the swarm produces internal forces in the object. With no leader robots, the position of the object is instead not controllable. On the other hand, one leader robot and nonzero internal forces on the object are sufficient to regulate both the position and orientation of the object to a desired value. If no internal forces are exerted on the object, at least two leader robots evenly spread around the object are necessary for the convergence of object position and orientation to a unique value in a ground system. For a floating swarm, at least three leader robots are instead necessary to achieve the same goal.

In addition to that, non-zero internal forces are enough to maintain the object attitude error bounded when external disturbances act on the system and there are no leader robots. To achieve robustness without compressing nor stretching the object (i.e., with zero internal forces in the object), at least two evenly distributed leader robots are necessary for a ground swarm, and three for a floating one.

\section{Modeling}

In this section, we describe a generic model and a compliant control approach for the multi-robot manipulation of an object (also referred to as the load in the following). The proposed model and control framework can be applied to ground, aerial and underwater vehicles as well.

To derive the model of the system, we first define an inertial frame $\mathcal{F}_{W}=$ $\left\{O_{W}, \boldsymbol{x}_{W}, \boldsymbol{y}_{W}, \boldsymbol{z}_{W}\right\}$ where $\left\{\boldsymbol{x}_{W}, \boldsymbol{y}_{W}, \boldsymbol{z}_{W}\right\}$ are its orthogonal unit axes. In particu- 


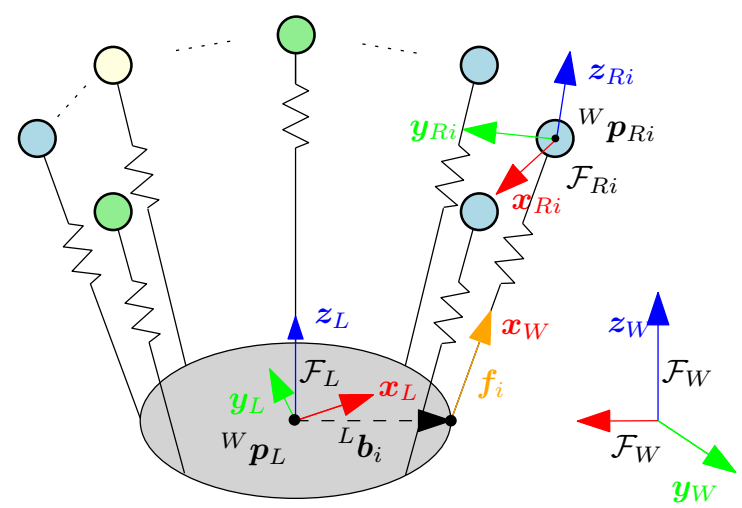

The behavior of each robot and the physical interconnection model are represented by springs.

Fig. 1: Representative illustration of the considered system and main variables. The load is represented by the gray disk. Each colored circle represents the end-effector of a robot. Different colors represent possible different classes of robots

lar, $\boldsymbol{z}_{W}$ is oriented in the opposite direction of the gravity vector. Then, we model the manipulated object as a rigid body subject to external forces, including the ones produced by the manipulating robots. We assume that the load has mass $m_{L} \in \mathbb{R}_{>0}$ and positive definite inertia matrix $\boldsymbol{J}_{L} \in \mathbb{R}^{3 \times 3}$. To describe its state, we define the frame $\mathcal{F}_{L}=\left\{O_{L}, \boldsymbol{x}_{L}, \boldsymbol{y}_{L}, \boldsymbol{z}_{L}\right\}$ rigidly attached to the load, where its origin, $O_{L}$, is centered on the load center of mass (CoM). $\left\{\boldsymbol{x}_{L}, \boldsymbol{y}_{L}, \boldsymbol{z}_{L}\right\}$ are its orthogonal unit axes. We refer to the position and orientation of $\mathcal{F}_{L}$ with respect to (w.r.t.) $\mathcal{F}_{W}$ by the vector ${ }^{W} \boldsymbol{p}_{L} \in \mathbb{R}^{3}$ and the rotation matrix ${ }^{1}{ }^{W} \boldsymbol{R}_{L} \in S O(3)$, respectively ${ }^{2}$. In the next sections, for the sake of description of the results, we shall parameterize the load orientation with the classical Euler angles following the convention $\mathrm{Z}-\mathrm{Y}-\mathrm{X}$, i.e., $\boldsymbol{\eta}=(\phi, \theta, \psi)$, called roll, pitch and yaw, respectively. We then define by $\dot{\boldsymbol{p}}_{L} \in \mathbb{R}^{3}$ the linear velocity of $\mathcal{F}_{L}$ and by ${ }^{L} \boldsymbol{\omega}_{L} \in \mathbb{R}^{3}$ the angular velocity of $\mathcal{F}_{L}$ w.r.t. $\mathcal{F}_{W}$ expressed in $\mathcal{F}_{L}$. Applying the standard Newton-Euler formalism we can write the dynamics of the load as:

$$
\begin{aligned}
m_{L} \ddot{\boldsymbol{p}}_{L} & =-m_{L} g \boldsymbol{z}_{W}-\boldsymbol{D}_{L} \dot{\boldsymbol{p}}_{L}+\boldsymbol{f}_{e} \\
\dot{\boldsymbol{R}}_{L} & =\boldsymbol{S}\left({ }^{L} \boldsymbol{\omega}_{L}\right) \boldsymbol{R}_{L} \\
\boldsymbol{J}_{L}{ }^{L} \dot{\boldsymbol{\omega}}_{L} & =-\boldsymbol{S}\left({ }^{L} \boldsymbol{\omega}_{L}\right) \boldsymbol{J}_{L}{ }^{L} \boldsymbol{\omega}_{L}-\boldsymbol{B}_{L}{ }^{L} \boldsymbol{\omega}_{L}+{ }^{L} \boldsymbol{\tau}_{e},
\end{aligned}
$$

where, $\boldsymbol{S}(\star)$ is the skew operator ${ }^{3}, g$ is the gravitational constant acceleration, $\boldsymbol{f}_{e}$ and ${ }^{L} \boldsymbol{\tau}_{e} \in \mathbb{R}^{3}$ are the sum of external forces and moments acting on the load with respect to $\mathcal{F}_{W}$ and $\mathcal{F}_{L}$, respectively. The positive definite matrices $\boldsymbol{D}_{L} \in \mathbb{R}^{3 \times 3}$ and $\boldsymbol{B}_{L} \in \mathbb{R}^{3 \times 3}$ model drag and friction effects. Note that the model can be simply modified for loads laying on the ground considering the following constraints:

$$
\boldsymbol{e}_{3}^{\top} \dot{\boldsymbol{p}}_{L}=0, \quad \boldsymbol{e}_{1}^{\top L} \boldsymbol{\omega}_{L}=0, \quad \boldsymbol{e}_{2}^{\top}{ }^{L} \boldsymbol{\omega}_{L}=0
$$

$1 S O(3)=\left\{\boldsymbol{R} \in \mathbb{R}^{3 \times 3} \mid \boldsymbol{R}^{\top} \boldsymbol{R}=\boldsymbol{I}_{3}\right\}$ where $\boldsymbol{I}_{i} \in \mathbb{R}^{i \times i}$ is the identity matrix of dimension $i$.

2 The left superscript indicates the reference frame. From now on, $\mathcal{F}_{W}$ is considered as reference frame when the superscript is omitted.

${ }^{3} \boldsymbol{S}(\star): \mathbb{R}^{3} \rightarrow \mathbb{R}^{3 \times 3}$ is such that $\boldsymbol{S}(\boldsymbol{x}) \boldsymbol{y}=\boldsymbol{x} \times \boldsymbol{y}$ for every $\boldsymbol{x} \in \mathbb{R}^{3}$ and $\boldsymbol{y} \in \mathbb{R}^{3}$ 
where $\boldsymbol{e}_{i} \in \mathbb{R}^{3}$ is the vector with a 1 in position $i$, and zero elsewhere.

The load is manipulated by a team of $N \in \mathbb{N}_{>2}$ robots (the case $N=2$ aerial robots have been already studied in Tognon et al. (2018)) equipped with a certain rigid or actuated tool ending in an end-effector whose position can be controlled by the robot. Looking at biological agents manipulating objects, such as bugs, birds, fishes or even human beings, they all share the type of actuation. In fact, they are actuated by muscles and tendons. The elastic components of such actuators (muscles and tendons) make the agents compliant with respect to external forces as the interaction forces with the environment. Such compliance has been proven to be essential for physical interaction tasks like pulling, pushing, sliding and peg-in-hole tasks. Inspired by the actuation compliance of biological agents, also robots that have to physically interact with the environment have been granted with such a property. Considering any type of ground, aerial or underwater robots, endowed with an interactive tool like a gripper, a cable, or a more complex articulated arm, the compliance of the end-effector can be virtual. Using a proper force-based controller, like the hybrid position-force controller, or the admittance and impedance controllers (see de Wit et al. (1996) and the citations therein), one can grant the robot the sought compliance. Those methods are based on the sensing of the interaction forces and the following reshaping of the system characteristics allowing it to behave like a different system with, e.g., more or less compliance. Recently, with the introduction of flexible joints, robots are endowed with mechanical compliance directly at the joint level.

Thanks to those compliant controllers, we can model the dynamics of the endeffector of the $i$-th robot as a simple mass-spring-damper system. A schematic representation of the considered system is given in Fig. 1. In particular, we define the frame $\mathcal{F}_{R i}=\left\{O_{i}, \boldsymbol{x}_{R i}, \boldsymbol{y}_{R i}, \boldsymbol{z}_{R i}\right\}$ rigidly attached to the $i$-th end-effector. Its configuration is then given by the position of $O_{i}$. In this work, we do not consider the end-effector orientation which is assumed to be the proper one to grasp the load according to the grasping method. We complete the $i$-th end-effector state with the linear velocity denoted by the vector $\dot{\boldsymbol{p}}_{R i} \in \mathbb{R}^{3}$. The dynamics of the endeffector of the $i$-th robot subject to a position control law, under the hypothesis of a perfect tracking, can be written as:

$$
\boldsymbol{M}_{i} \ddot{\boldsymbol{p}}_{R i}=\boldsymbol{f}_{C i}
$$

where $\boldsymbol{f}_{C i}$ is the "control" force provided by each robot. The additional control input $\boldsymbol{f}_{C i}$ is then set as:

$$
\boldsymbol{f}_{C i}=-\boldsymbol{f}_{i}-\boldsymbol{B}_{i} \dot{\boldsymbol{p}}_{R i}-\boldsymbol{K}_{i}\left(\boldsymbol{p}_{R i}-\boldsymbol{p}_{R i}^{d}\right)+\boldsymbol{\pi}_{i},
$$

where the three positive definite symmetric matrices $\boldsymbol{M}_{i}, \boldsymbol{B}_{i}, \boldsymbol{K}_{i} \in \mathbb{R}^{3 \times 3}$ are the virtual inertia of the robot, the virtual damping, and the stiffness of a virtual spring attached to the robot. $f_{i} \in \mathbb{R}^{3}$ is the sensed force of interaction with the environment, e.g., the load. Finally, $\boldsymbol{\pi}_{i} \in \mathbb{R}^{3}$ is the desired interaction force, expressed w.r.t. $\mathcal{F}_{W}$. In the following, we shall show that $\boldsymbol{\pi}_{i}$, setting non-zero internal forces on the load ${ }^{4}$, plays an important role in shaping the system equilibria and its stability as well.

\footnotetext{
4 Forces that do not result in a motion of the object, nor compensate any external wrench. For a more complete description, the reader can refer to Prattichizzo and Trinkle (2008)
} 
In this work, we shall focus only on the motion of the robot end-effector, assuming that the low-level controller makes the model (4) always valid, at least in the working conditions. From now on, we shall refer to the $i$-th end-effector as the $i$-th robot.

We consider the common case in which the robots are attached to the load in an evenly distributed way along a circle centered on $O_{L}$ with radius $b \in \mathbb{R}_{>0}$. In particular, assuming that the $i$-th robot is in contact with the load at the point $B_{i}$, described by the vector ${ }^{L} \boldsymbol{b}_{i} \in \mathbb{R}^{3}$, we assume without loss of generality that:

$$
{ }^{L} \boldsymbol{b}_{i}=b\left[\begin{array}{lll}
\cos \theta_{i} & \sin \theta_{i} & 0
\end{array}\right]^{\top}=:\left[\begin{array}{lll}
x_{i} & y_{i} & 0
\end{array}\right]^{\top},
$$

where $\theta_{i}=(i-1) 2 \pi / N$. It will be clear in the following that this configuration allows to equally share the effort among all the robots.

The contact force $\boldsymbol{f}_{i}$ between the load and the robot end-effector can be modeled as a function of $\boldsymbol{p}_{R i}$ and the corresponding contact point expressed in $\mathcal{F}_{W}$, $\boldsymbol{b}_{i}=\boldsymbol{p}_{L}+\boldsymbol{R}_{L}{ }^{L} \boldsymbol{b}_{i}$. In other words, $\boldsymbol{f}_{i}=\boldsymbol{h}\left(\boldsymbol{p}_{R i}, \boldsymbol{p}_{L}, \boldsymbol{R}_{L}\right)$. Assuming a friction-less contact, we can model it as an elastic force:

$$
\boldsymbol{f}_{i}=q_{i} \boldsymbol{n}_{i}, \quad \text { with } \quad q_{i}=k_{i}\left(\left\|\boldsymbol{l}_{i}\right\|-l_{0 i}\right),
$$

where $\boldsymbol{l}_{i}=\boldsymbol{p}_{R i}-\left(\boldsymbol{p}_{L}+\boldsymbol{R}_{L}{ }^{L} \boldsymbol{b}_{i}\right)$ is the contact displacement, $\boldsymbol{n}_{i}=\boldsymbol{l}_{i} /\left\|\boldsymbol{l}_{i}\right\|$ is the force direction described by a normalized vector, $q_{i}$ is the intensity of the contact force, $k_{i} \in \mathbb{R}_{>0}$ is the elastic coefficient, and $l_{0 i} \in \mathbb{R}$ is a displacement offset. Note that this model comprehends also the interesting case in which each robot is connected to the load by cables. In this particular case, $k_{i}$ is the cable stiffness and $l_{0 i}$ is its nominal length. Equation (6) can be slightly modified to consider the case of a slack cable:

$$
q_{i}=\left\{\begin{array}{ll}
k_{i}\left(\left\|\boldsymbol{l}_{i}\right\|-l_{0 i}\right) & \text { if }\left\|\boldsymbol{l}_{i}\right\|-l_{0 i}>0 \\
0 & \text { otherwise }
\end{array} .\right.
$$

We remark that other contact models can be considered to characterize phenomena like friction cone, dissipation, etc. (see Machado et al. (2012); Anitescu and Potra (1997)).

Finally, gathering all the contact forces between the robots and the load in the vector $\boldsymbol{f}=\left[\boldsymbol{f}_{1}^{\top} \ldots \boldsymbol{f}_{N}^{\top}\right]^{\top} \in \mathbb{R}^{3 N}$, we can write the total force and moment applied to the load. This helps to complete the load closed-loop dynamics in (1) as:

$$
\left[\begin{array}{c}
\boldsymbol{f}_{e} \\
{ }^{L} \boldsymbol{\tau}_{e}
\end{array}\right]=\underbrace{\left[\begin{array}{ccc}
\boldsymbol{I}_{3} & \ldots & \boldsymbol{I}_{3} \\
\boldsymbol{S}\left({ }^{L} \boldsymbol{b}_{1}\right) \boldsymbol{R}_{L}^{\top} & \ldots & \boldsymbol{S}\left({ }^{L} \boldsymbol{b}_{N}\right) \boldsymbol{R}_{L}^{\top}
\end{array}\right]}_{\boldsymbol{G}\left(\boldsymbol{R}_{L}\right)} \boldsymbol{f} .
$$

Remark 1 The model presented above is really general and can be applied to any ground, aerial or underwater swarm of robots manipulating a rigid load. The agents can even be biological, under the sole assumption that the end-effector of each agent behaves as a mass-spring-damper system, (4). As highlighted before, this is the most common case for biological agents thanks to their natural compliant actuation. 


\section{Problem formulation}

The aim of this work is to analyze and understand which are the conditions on the robots and their control parameters in order to stabilize the load in a desired configuration, $\boldsymbol{q}_{L}^{d}=\left(\boldsymbol{p}_{L}^{d}, \boldsymbol{R}_{L}^{d}\right)$, considering both position and orientation. Although it is not very common in the literature of collaborative transportation, here we focus on the precise regulation of the load attitude as well. In fact, in many practical applications, like assembly, the simultaneous control of the position and orientation of the load is very important. Therefore, keeping the load in the horizontal position is here the main purpose, i.e., $\boldsymbol{R}_{L}^{d}=\boldsymbol{R}_{\boldsymbol{z}_{W}}\left(\psi^{d}\right)$ for every ${ }^{5} \psi^{d} \in[0,2 \pi]$. Without loss of generality, simply applying an opportune rotation of $\mathcal{F}_{W}$, we can set $\boldsymbol{R}_{L}^{d}=\boldsymbol{I}_{3}$.

In our previous works (Gabellieri et al., 2018; Tognon et al., 2018), it has been demonstrated that the proposed compliant control law, in association with a leader-follower control scheme, represents an effective strategy to control the load position and orientation without relying on explicit communication. Therefore, with this work, we intend to extend the previous results toward a more complete analysis in terms of:

1. class of system, including both ground and floating systems, and analyzing their differences;

2. formal equilibria and stability analysis in the case of $N$ robots;

3. influence of the internal forces not only on the convergence to a desired object configuration but also on the disturbance rejection capabilities;

4. role of the number of leader robots.

Assumption 1 the robots are attached to the load in an evenly distributed way along a circle centered in $O_{L}$;

Assumption 2 the leader is the only robot that is aware of its own desired position based on the desired position and orientation of the load. The followers might know the desired orientation of the load, as it will be more clear in the following. However, they are not attracted to any desired position;

Assumption 3 absence of explicit communication;

Assumption 4 perfect position tracking is supposed for all the robots.

Several numerical simulations for different conditions of interest validate our formal analysis, allowing us to empirically draw extra interesting conclusions from the observation of the obtained results.

\section{Equilibria and Stability Analysis}

The system is said to be in static equilibrium when there exists a set of forces that brings the velocities and accelerations to zero. Adding this condition to (1), we simply have that $\left[\begin{array}{ll}m_{L} g \boldsymbol{z}_{W}^{\top} & \mathbf{0}_{1 \times 3}^{\top}\end{array}\right]^{\top}=\left[\begin{array}{ll}\boldsymbol{f}_{e}^{\top} & { }^{L} \boldsymbol{\tau}_{e}^{\top}\end{array}\right]^{\top}$. Replacing it into (8) we obtain the following equilibrium equation

$$
\left[m_{L} g \boldsymbol{z}_{W}^{\top} \mathbf{0}_{1 \times 3}^{\top}\right]^{\top}=\boldsymbol{G}\left(\boldsymbol{R}_{L}\right) \boldsymbol{f}
$$

\footnotetext{
${ }^{5} \boldsymbol{R}_{\boldsymbol{z}_{W}}(\star) \in S O(3)$ describes a rotation along the axes $\boldsymbol{z}_{W}$ about the angle $\star$.
} 
Given the desired load configuration $\left(\boldsymbol{p}_{L}^{d}, \boldsymbol{R}_{L}^{d}\right)$ and resolving (9) for $\boldsymbol{f}$, we can obtain the forces that the robots should apply such that $\boldsymbol{R}_{L}^{d}$ is of equilibrium:

$$
\boldsymbol{f}=\boldsymbol{G}\left(\boldsymbol{R}_{L}^{d}\right)^{\dagger}\left[m_{L} g \boldsymbol{z}_{W}^{\top} 0_{1 \times 3}\right]^{\top}+\boldsymbol{t},
$$

where $^{\dagger}$ indicates a right (pseudo)inverse, and $\boldsymbol{t}=\left[\begin{array}{llll}\boldsymbol{t}_{1}^{\top} & \boldsymbol{t}_{2}^{\top} & \ldots & \boldsymbol{t}_{N}^{\top}\end{array}\right]^{\top} \in \operatorname{null}\left(\boldsymbol{G}\left(\boldsymbol{R}_{L}^{d}\right)\right)$ $\subset \mathbb{R}^{3 N}$ is a vector containing the robot forces that produce internal forces on the object. It is easy to verify that a particular solution for which the efforts among the robots are balanced is:

$$
\boldsymbol{f}_{i}=\overline{\boldsymbol{f}}_{i}\left(\boldsymbol{q}_{L}^{d}, f_{\mathrm{int}}\right):=\frac{m_{L} g}{N} \boldsymbol{z}_{W}+f_{\mathrm{int}} \boldsymbol{R}_{L}^{d} \frac{{ }^{L} \boldsymbol{b}_{i}}{b},
$$

where $f_{\text {int }} \in \mathbb{R}$ is referred to as the internal force intensity and is, indeed, the same for each robot. Notice that:

- If $f_{\text {int }}=0$, the interaction forces are vertical (null in the case of ground systems). For the case of a cable-suspended load, the cables would be vertical.

- If $f_{\text {int }}>0$, the interaction forces are oriented radially and outward with respect to the load CoM. In this case, the robots tend to stretch the object producing an internal tension.

- If $f_{\text {int }}<0$, the interaction forces are oriented radially and inward with respect to the load CoM. In this case, the robots tend to push the object producing an internal compression.

From simple kinematics and using (6), we can find the position of the robots such that $\boldsymbol{p}_{L}^{d}$ is of equilibrium:

$$
\boldsymbol{p}_{R i}^{d}\left(\boldsymbol{q}_{L}^{d}, f_{\mathrm{int}}\right)=\boldsymbol{p}_{L}^{d}+\boldsymbol{R}_{L}^{d}{ }^{L} \boldsymbol{b}_{i}+\left(\frac{\left\|\overline{\boldsymbol{f}}_{i}\right\|}{k_{i}}+l_{0 i}\right) \frac{\overline{\boldsymbol{f}}_{i}}{\left\|\overline{\boldsymbol{f}}_{i}\right\|}
$$

Finally, imposing the equilibrium condition to (4) $\left(\ddot{\boldsymbol{p}}_{R i}=\dot{\boldsymbol{p}}_{R i}=\mathbf{0}\right)$ and considering (11), we can find the forcing input for each robot that makes the desired load configuration $\boldsymbol{q}_{L}^{d}$ an equilibrium. In particular, it has to be that $\boldsymbol{\pi}_{i}=\boldsymbol{\pi}_{A i}^{d}\left(\boldsymbol{q}_{L}^{d}, f_{\mathrm{int}}\right)=\overline{\boldsymbol{f}}_{i}\left(\boldsymbol{q}_{L}^{d}, f_{\mathrm{int}}\right)$.

Under the assumptions 2 and 3 , for every follower robot, it holds that $\boldsymbol{K}_{i}=$ $\mathbf{0}_{3 \times 3}$. Notice that in order to compute the forcing input as in (11), the followers need to know the desired orientation of the load. By contrast, if no internal force reference is set, they only require the knowledge of the load mass (and only for a floating load). In this way, the followers will simply follow the motion of the leader, trying to match the desired interaction force with the sensed one, damping its motion, and so the one of the load, at the same time.

Given a desired load equilibrium $\boldsymbol{q}_{L}^{d}$, and a value of the internal force intensity $f_{\text {int }}$, every robot applies the forcing input $\boldsymbol{\pi}_{A i}^{d}\left(\boldsymbol{q}_{L}^{d}, f_{\text {int }}\right)$. Under this condition, it is not granted that $\boldsymbol{q}_{L}^{d}$ is the unique load configuration of equilibrium. To find all the equilibria under the forcing input $\boldsymbol{\pi}_{A i}^{d}\left(\boldsymbol{q}_{L}^{d}, f_{\text {int }}\right)$ we need to find $\boldsymbol{p}_{L}$ and $\boldsymbol{R}_{L}$ that are solutions of (4) and (9) when $\boldsymbol{\pi}_{i}=\boldsymbol{\pi}_{A i}^{d}$, and velocities and accelerations are equal to zero (equilibrium condition). From (4) it has to be:

$$
\mathbf{0}=-\boldsymbol{f}_{i}-\boldsymbol{K}_{i}\left(\boldsymbol{p}_{R i}-\boldsymbol{p}_{R i}^{d}\right)+\boldsymbol{\pi}_{A i}^{d} .
$$


Replacing (13) into (9) and considering the first three rows, we obtain:

$$
m_{L} g \boldsymbol{z}_{W}=\sum_{i=1}^{N}\left(-\boldsymbol{K}_{i}\left(\boldsymbol{p}_{R i}-\boldsymbol{p}_{R i}^{d}\right)+\frac{m_{L} g}{N} \boldsymbol{z}_{W}+f_{\mathrm{int}} \boldsymbol{R}_{L}^{d} \frac{{ }^{L} \boldsymbol{b}_{i}}{b}\right),
$$

which implies $\sum_{i=1}^{N}-\boldsymbol{K}_{i}\left(\boldsymbol{p}_{R i}-\boldsymbol{p}_{R i}^{d}\right)=\mathbf{0}$. In fact, it is easy to verify that $\sum_{i=1}^{N}{ }^{L} \boldsymbol{b}_{i}=$ $\mathbf{0}$. Notice that in the case of a single leader (1st robot), we have $\boldsymbol{K}_{1} \neq \mathbf{0}$, while $\boldsymbol{K}_{i}=\mathbf{0} \forall i \neq 1$. In this case, $\boldsymbol{p}_{R 1}=\boldsymbol{p}_{R 1}^{d}$. Considering the last three rows of (9), and recalling that $\boldsymbol{R}_{L}^{d}=\boldsymbol{I}_{3}$, we obtain:

$$
\mathbf{0}=\sum_{i=1}^{N}{ }^{L} \boldsymbol{b}_{i} \times \boldsymbol{R}_{L}^{\top} \overline{\boldsymbol{f}}_{i}=\frac{f_{\text {int }}}{b} \sum_{i=1}^{N}{ }^{L} \boldsymbol{b}_{i} \times \boldsymbol{R}_{L}^{\top}{ }^{L} \boldsymbol{b}_{i}+\sum_{i=1}^{N}{ }^{L} \boldsymbol{b}_{i} \times \boldsymbol{R}_{L}^{\top} \frac{m_{L} g}{N} \boldsymbol{z}_{W} .
$$

Noticing that $b \neq 0$, and $\sum_{i=1}^{N}{ }^{L} \boldsymbol{b}_{i}=\mathbf{0},(15)$ yields:

$$
\mathbf{0}=f_{\text {int }} \sum_{i=1}^{N}{ }^{L} \boldsymbol{b}_{i} \times \boldsymbol{R}_{L}^{\top}{ }^{L} \boldsymbol{b}_{i}
$$

If $f_{\text {int }}=0$, then (16) holds for every $\boldsymbol{R}_{L} \in S O(3)$. Instead, if $f_{\text {int }} \neq 0$, remembering definition (5), and defining $r_{i j} \in \mathbb{R}$ as the element of $\boldsymbol{R}_{L}$ in position $(i, j),(16)$ can be rewritten as:

$$
\begin{cases}\sum_{i=1}^{N} r_{31} y_{i} x_{i}+r_{32} y_{i}^{2} & =0 \\ \sum_{i=1}^{N}-r_{31} x_{i}^{2}-r_{32} x_{i} y_{i} & =0 \\ \sum_{i=1}^{N} x_{i}\left(-y_{i} r_{11}+x_{i} r_{12}\right)+y_{i}\left(-y_{i} r_{21}+x_{i} r_{22}\right) & =0\end{cases}
$$

With the definition (5) in mind and $N>2$, it is easy to verify the following properties:

$$
\sum_{i=1}^{N} x_{i}=0, \quad \sum_{i=1}^{N} y_{i}=0, \quad \sum_{i=1}^{N} x_{i} y_{i}=0, \quad \sum_{i=1}^{N} x_{i}^{2}=\sum_{i=1}^{N} y_{i}^{2}=c>0 .
$$

Using the previous results into (17), we have that $r_{31}=r_{32}=0$ and $r_{21}=r_{12}$. Imposing the rotation matrix conditions, i.e., $\boldsymbol{R}_{L}^{\top} \boldsymbol{R}_{L}=\boldsymbol{I}_{3}$ and $\operatorname{det}\left(\boldsymbol{R}_{L}\right)=1$, we finally obtain $\boldsymbol{R}_{L}=\operatorname{diag}(1,1,1)$ or $\boldsymbol{R}_{L}=\operatorname{diag}(-1,-1,1)$, which means $\boldsymbol{R}_{L}=$ $\boldsymbol{R}_{\boldsymbol{z}}(k \pi) \boldsymbol{R}_{L}^{d}$, with $k \in \mathbb{N}$. The position of the load can be then computed by simple kinematics:

$$
\boldsymbol{p}_{L}=\boldsymbol{p}_{R 1}-\boldsymbol{R}_{L}{ }^{L} \boldsymbol{b}_{1}-\left(\frac{\left\|\overline{\boldsymbol{f}}_{1}\right\|}{k_{1}}+l_{01}\right) \frac{\overline{\boldsymbol{f}}_{1}}{\left\|\overline{\boldsymbol{f}}_{1}\right\|} .
$$

Summarizing, given the closed-loop system under the forcing input $\boldsymbol{\pi}_{A i}^{d}\left(\boldsymbol{q}_{L}^{d}, f_{\text {int }}\right)$, all the equilibrium load configurations $\left(\boldsymbol{p}_{L}, \boldsymbol{R}_{L}\right)$ are such that $\boldsymbol{p}_{L}$ is computed as in (19) and:

$-\boldsymbol{R}_{L} \in S O(3)$ if $f_{\text {int }}=0$

$-\boldsymbol{R}_{L}=\boldsymbol{R}_{\boldsymbol{z}}(k \pi) \boldsymbol{R}_{L}^{d}$, with $k \in \mathbb{N}$, if $f_{\text {int }} \neq 0$. 
Remark 2 Similarly to the case with two robots in Tognon et al. (2018), when $f_{\text {int }}=0$, an infinite number of equilibrium configurations exists. For each of them, the anchoring point position of the leader to the load is constant, while the attitude of the load can be any. By contrast, when $f_{\text {int }} \neq 0$ there are only two equilibrium configurations. One is equal to the desired one, while the other has the load turned by $\pi$ about a vertical axis passing through the leader anchoring point. We shall show that for $f_{\text {int }}>0$, the first is asymptotically stable while the second is unstable. Therefore selecting $f_{\text {int }}>0$ is the most suitable choice.

Aiming to prove the stable/unstable nature of the equilibria previously identified, we define by $\boldsymbol{x}=\left(\boldsymbol{p}_{R 1}, \ldots, \boldsymbol{p}_{R N}, \boldsymbol{p}_{L}, \boldsymbol{R}_{L}, \dot{\boldsymbol{p}}_{R 1}, \ldots, \dot{\boldsymbol{p}}_{R N}, \dot{\boldsymbol{p}}_{L},{ }^{L} \boldsymbol{\omega}_{L}\right)$ the state of the system, and by $\mathbb{X}$ the relative state space. We then define the set of zero velocity states containing all the equilibrium configurations under a specific forcing input $\boldsymbol{\pi}_{A i}^{d}\left(\boldsymbol{q}_{L}^{d}, f_{\text {int }}\right)$ as $\mathcal{X}\left(f_{\text {int }}, \boldsymbol{q}_{L}^{d}\right)=\left\{\boldsymbol{x} \in \mathbb{X} \mid \boldsymbol{p}_{L}, \boldsymbol{R}_{L}\right.$ respect (19) and (16), $\boldsymbol{p}_{R i}$ is given by inverse kinematics, and $\left.\dot{\boldsymbol{p}}_{R 1}=\ldots=\dot{\boldsymbol{p}}_{R N}=\dot{\boldsymbol{p}}_{L}={ }^{L} \boldsymbol{\omega}_{L}=\mathbf{0}\right\}$. In addition, for $f_{\text {int }} \neq 0$, we define $\boldsymbol{x}^{\prime}\left(f_{\text {int }}, \boldsymbol{q}_{L}^{d}\right) \in \mathcal{X}\left(f_{\text {int }}, \boldsymbol{q}_{L}^{d}\right)$ and $\boldsymbol{x}^{\prime \prime}\left(f_{\text {int }}, \boldsymbol{q}_{L}^{d}\right) \in \mathcal{X}\left(f_{\text {int }}, \boldsymbol{q}_{L}^{d}\right)$ such that $\boldsymbol{R}_{L}=\boldsymbol{R}_{L}^{d}$ and $\boldsymbol{R}_{L}=\boldsymbol{R}_{\boldsymbol{z}}(\pi) \boldsymbol{R}_{L}^{d}$, respectively. Notice that for $f_{\text {int }} \neq 0$, $\mathcal{X}\left(f_{\text {int }}, \boldsymbol{q}_{L}^{d}\right)=\left\{\boldsymbol{x}^{\prime}\left(f_{\mathrm{int}}, \boldsymbol{q}_{L}^{d}\right), \boldsymbol{x}^{\prime \prime}\left(f_{\text {int }}, \boldsymbol{q}_{L}^{d}\right)\right\}$. At this point, the next theorem can be formulated.

Theorem 1 Given a desired load configuration $\boldsymbol{q}_{L}^{d}$ and considering the closed-loop system (1) and (4) under the forcing input $\boldsymbol{\pi}_{A i}^{d}\left(\boldsymbol{q}_{L}^{d}, f_{\text {int }}\right)$ :

- every $\boldsymbol{x} \in \mathcal{X}\left(f_{\text {int }}, \boldsymbol{q}_{L}^{d}\right)$ is stable for $f_{\text {int }}=0$;

$-\boldsymbol{x}^{\prime}\left(f_{\mathrm{int}}, \boldsymbol{q}_{L}^{d}\right)$ is asymptotically stable if $f_{\mathrm{int}}>0$;

$-\boldsymbol{x}^{\prime \prime}\left(f_{\mathrm{int}}, \boldsymbol{q}_{L}^{d}\right)$ is unstable if $f_{\mathrm{int}}>0$;

$-\boldsymbol{x}^{\prime}\left(f_{\mathrm{int}}, \boldsymbol{q}_{L}^{d}\right)$ is unstable if $f_{\mathrm{int}}<0$;

$-\boldsymbol{x}^{\prime \prime}\left(f_{\text {int }}, \boldsymbol{q}_{L}^{d}\right)$ is asymptotically stable if $f_{\text {int }}<0$.

Proof Let us consider the following Lyapunov candidate:

$$
\begin{aligned}
V(\boldsymbol{x})= & \frac{1}{2}\left(\sum_{i=1}^{N} \dot{\boldsymbol{p}}_{R i}^{\top} \boldsymbol{M}_{i} \dot{\boldsymbol{p}}_{R i}+\boldsymbol{e}_{R i}^{\top} \boldsymbol{K}_{i} \boldsymbol{e}_{R i}+k_{i}\left(\left\|\boldsymbol{l}_{i}\right\|-l_{0 i}\right)^{2}-2 \boldsymbol{l}_{i}^{\top} \overline{\boldsymbol{f}}_{i}\right)+ \\
& +\left(N f_{\text {int }} b-\sum_{i=1}^{N} \overline{\boldsymbol{f}}_{i}^{\top} \boldsymbol{R}_{L}{ }^{L} \boldsymbol{b}_{i}\right)+m_{L} \dot{\boldsymbol{p}}_{L}^{\top} \dot{\boldsymbol{p}}_{L}+{ }^{L} \boldsymbol{\omega}_{L}^{\top} \boldsymbol{J}_{L}{ }^{L} \boldsymbol{\omega}_{L}+V_{0},
\end{aligned}
$$

where $V_{0} \in \mathbb{R}_{\geq 0}$ and $\boldsymbol{e}_{R i}=\boldsymbol{p}_{R i}^{d}-\boldsymbol{p}_{R i}$. Note that for $i \neq 1$ one can set $\boldsymbol{e}_{R i}$ arbitrarily, since the terms multiply $\boldsymbol{K}_{i}=\mathbf{0}$. For an opportune choice of $V_{0}, V(\boldsymbol{x})$ is a positive definite, continuously differentiable function in the domain of interest for which we have that $\boldsymbol{x}_{\text {min }}\left(\boldsymbol{x}_{\text {min }}=\operatorname{argmin}_{\boldsymbol{x}} V(\boldsymbol{x})\right)$ is such that $\boldsymbol{x}_{\text {min }} \in \mathcal{X}\left(0, \boldsymbol{q}_{L}^{d}\right)$ and $\boldsymbol{x}_{\text {min }}=\boldsymbol{x}^{\prime}\left(f_{\text {int }}, \boldsymbol{q}_{L}^{d}\right)$ for $f_{\text {int }}>0$. The proof of this result has been derived following similar reasonings done in Tognon et al. (2018), and here it is omitted for the sake of brevity. Notice that $V(\boldsymbol{x})=0$ for all $\boldsymbol{x} \in \mathcal{X}\left(0, \boldsymbol{q}_{L}^{d}\right)$ and $\boldsymbol{x}=\boldsymbol{x}^{\prime}\left(f_{\text {int }}, \boldsymbol{q}_{L}^{d}\right)$ for $f_{\text {int }}>0$. Please see Tognon et al. (2018) for further details.

Let us now compute the time derivative of (20):

$$
\begin{aligned}
\dot{V}(\boldsymbol{x})= & \left(\sum_{i=1}^{N} \dot{\boldsymbol{p}}_{R i}^{\top} \boldsymbol{M}_{i} \ddot{\boldsymbol{p}}_{R i}+\boldsymbol{e}_{R i}^{\top} \boldsymbol{K}_{i} \dot{\boldsymbol{e}}_{R i}+i_{i}^{\top} \boldsymbol{f}_{i}-\dot{\boldsymbol{l}}_{i}^{\top} \overline{\boldsymbol{f}}_{i}+\overline{\boldsymbol{f}}_{i}^{\top} \boldsymbol{R}_{L} \boldsymbol{S}\left({ }^{L} \boldsymbol{\omega}_{L}\right)^{L} \boldsymbol{b}_{i}\right) \\
& +m_{L} \dot{\boldsymbol{p}}_{L}^{\top} \ddot{\boldsymbol{p}}_{L}+{ }^{L} \boldsymbol{\omega}_{L}^{\top} \boldsymbol{J}_{L}{ }^{L} \dot{\boldsymbol{\omega}}_{L},
\end{aligned}
$$


where we used the fact that $\frac{d}{d t}\left(\frac{1}{2} k_{i}\left(\left\|\boldsymbol{l}_{i}\right\|-l_{0 i}\right)^{2}\right)=\boldsymbol{i}_{i}^{\top} \boldsymbol{f}_{i}$. Replacing (1) and (4) we obtain:

$$
\begin{aligned}
\dot{V}(\boldsymbol{x})= & \left(\sum_{i=1}^{N} \dot{\boldsymbol{p}}_{R i}^{\top}\left(-\boldsymbol{f}_{i}-\boldsymbol{B}_{i} \dot{\boldsymbol{p}}_{R i}-\boldsymbol{K}_{i} \boldsymbol{e}_{R i}+\overline{\boldsymbol{f}}_{i},\right)+\dot{\boldsymbol{p}}_{R i}^{\top} \boldsymbol{K}_{i} \boldsymbol{e}_{R i}+\dot{\boldsymbol{l}}_{i}^{\top}\left(\boldsymbol{f}_{i}-\overline{\boldsymbol{f}}_{i}\right)+\right. \\
& \left.+\overline{\boldsymbol{f}}_{i}^{\top} \boldsymbol{R}_{L} \boldsymbol{S}\left({ }^{L} \boldsymbol{\omega}_{L}\right){ }^{L} \boldsymbol{b}_{i}\right)+\dot{\boldsymbol{p}}_{L}^{\top}\left(-m_{L} g \boldsymbol{z}_{W}-\boldsymbol{D}_{L} \dot{\boldsymbol{p}}_{L}+\sum_{i=1}^{N} \boldsymbol{f}_{i}\right)+ \\
& +{ }^{L} \boldsymbol{\omega}_{L}^{\top} \boldsymbol{J}_{L}\left(-\boldsymbol{S}\left({ }^{L} \boldsymbol{\omega}_{L}\right) \boldsymbol{J}_{L}{ }^{L} \boldsymbol{\omega}_{L}-\boldsymbol{B}_{L}{ }^{L} \boldsymbol{\omega}_{L}+\sum_{i=1}^{N} \boldsymbol{S}\left({ }^{L} \boldsymbol{b}_{i}\right) \boldsymbol{R}_{L} \boldsymbol{f}_{i}\right),
\end{aligned}
$$

Noticing that $\dot{\boldsymbol{l}}_{i}=\dot{\boldsymbol{p}}_{R i}-\dot{\boldsymbol{p}}_{L}-\boldsymbol{R} \boldsymbol{S}\left({ }^{L} \boldsymbol{\omega}_{L}\right){ }^{L} \boldsymbol{b}_{i}$ and $\sum_{i=1}^{N} \overline{\boldsymbol{f}}_{i}=-m_{L} g \boldsymbol{z}_{W}$, after few algebraic computations we get:

$$
\dot{V}(\boldsymbol{x})=\sum_{i=1}^{N}-\dot{\boldsymbol{p}}_{R i}^{\top} \boldsymbol{B}_{i} \dot{\boldsymbol{p}}_{R i}-\dot{\boldsymbol{p}}_{L}^{\top} \boldsymbol{D}_{L} \dot{\boldsymbol{p}}_{L}-{ }^{L} \boldsymbol{\omega}_{L}^{\top} \boldsymbol{B}_{L}{ }^{L} \boldsymbol{\omega}_{L},
$$

that is clearly negative semidefinite. In particular $\dot{V}(\boldsymbol{x})=0$ for all $\boldsymbol{x} \in \mathcal{E}=\{\boldsymbol{x} \in$ $\left.\mathbb{X} \mid \dot{\boldsymbol{p}}_{R i}=\mathbf{0} \forall i, \dot{\boldsymbol{p}}_{L}=\mathbf{0},{ }^{L} \boldsymbol{\omega}_{L}=\mathbf{0}\right\}$. Based on the LaSalle's invariance principle and the Chetaev's theorem (see Khalil (2001)), with only minor modifications with respect to what previously done in Tognon et al. (2018), the stability nature of the equilibria according to $f_{\text {int }}$ can be proved.

Let us define the input-output pair $(\boldsymbol{u}, \boldsymbol{y})$, where $\boldsymbol{u}$ and $\boldsymbol{y}$ are, respectively, the stack vectors of $\boldsymbol{p}_{R i}^{d}$ and $\dot{\boldsymbol{p}}_{R i}$ for all $i$. Using the same Lyapunov function (20), it is easy to show that even with a time-varying desired trajectory for the leader robot, the system is output-strictly passive w.r.t. the storage function (20) and the input-output pair $(\boldsymbol{u}, \boldsymbol{y})$. The proof follows the same reasonings done in Tognon et al. (2018).

Remark 3 Once the desired pose of the load is decided, it is advisable to choose $f_{\text {int }}>0$. In this condition, setting the forcing inputs as $\boldsymbol{\pi}_{A i}^{d}$, the robots will steer the load to the desired configuration preserving the stability without the need of sending data to each other. Moreover, by virtue of the passivity, a certain robustness with respect to uncertainties and external disturbances can be guaranteed by the controlled system. Finally, it has to be noticed that implicit communication has been exploited in order to execute the cooperative task. Indeed, the robots can exchange and feel the forces from the cables and the object.

\section{Numerical Analysis}

We present here the validation of the presented theoretical results through numerical simulations. The effect of changing the number of leader robots is also investigated. The simulations are carried out both in nominal and far-from-ideal conditions.

The simulated system has the following characteristics. The load is a rigid body with mass $m_{L}=5 \mathrm{~kg}$ and inertia matrix $\boldsymbol{J}_{L}=\boldsymbol{I}_{3} \mathrm{~kg} \mathrm{~m}^{2}$. The contact stiffness is 
set to $900 \mathrm{Nm}$. The leader robots gains are: $\boldsymbol{M}=0.5 \boldsymbol{I}_{3} \mathrm{~kg}, \boldsymbol{B}=100 \boldsymbol{I}_{3} \mathrm{~N} \mathrm{~s} \mathrm{~m}^{-1}$ and $\boldsymbol{K}_{1}=1000 \boldsymbol{I}_{3} \mathrm{~N} \mathrm{~m}^{-1}$, and the follower robots ones are: $\boldsymbol{M}=0.01 \boldsymbol{I}_{3} \mathrm{~kg}, \boldsymbol{B}=$ $0.15 \boldsymbol{I}_{3} \mathrm{~N} \mathrm{~s} \mathrm{~m}^{-1}$ and $\boldsymbol{K}_{1}=0 \boldsymbol{I}_{3} \mathrm{~N} \mathrm{~m}^{-1}$. For the simulations with a single leader, we considered a swarm composed by a variable number of robots, randomly varying between 2 to 50 . On the other hand, for the simulations with a number of leaders different from one, we considered swarms composed of 12 robots. In particular, we have that the radius of the circle around which the robots are attached is $b=2.5 \mathrm{~m}$. The desired attitude of the object is described by desired roll, pitch and yaw angles $\theta^{d}=\phi^{d}=\psi^{d}=0$. We will refer to this attitude as $\boldsymbol{\eta}_{0}=(0,0,0)$. Let us also define $\boldsymbol{\eta}_{\pi}=(0,0, \pm \pi)$. We remark that the chosen desired configuration, in which the object is horizontal, is indeed of the uttermost practical relevance, as, e.g., in assembly applications. Note that, with a rotation of $\mathcal{F}_{W}$ around $\boldsymbol{z}_{W}$ by $\psi^{d}$, the results can be extended for any value of $\psi^{d}$.

We remark that only some simulation results are reported here for the sake of readability and compactness. Additional results can be found in the Online supplementary material.

\subsection{Convergence analysis}

In this section, we want to show the convergence of the load configuration to a certain static equilibrium, with respect to i) the value of $f_{\text {int }}$; ii) the number of leader robots. If there is at least one leader, robot 1 is always considered a leader.

In order to investigate the capability of the system to bring the load to a specific configuration of equilibrium, i.e., its stability, we initialize the system in several different initial conditions. The initial conditions are characterized by random values of $\psi, \theta$, and $\phi$ (the last two only in the case of floating systems) between $-\pi / 4 \mathrm{rad}$ and $\pi / 4 \mathrm{rad})$.

The number of leader robots ranges between 0 and 3 . For each number of leader robots, tests have been carried out with the following values of $f_{\text {int }}$ :

1) $f_{\text {int }}=0 \mathrm{~N}$,

2) $f_{\text {int }}=0.8 \mathrm{~N}(>0)$,

3) $f_{\text {int }}=-0.8 \mathrm{~N}(<0)$.

Note that, in the cases 2) and 3), the value of $f_{\text {int }}$ is equal to $\pm 0.016 \%$ of the total weight-force of the load. For each one of the cases obtained by a combination of the number of leader robots and value of $f_{\text {int }}, 10$ trajectories are displayed, each one starting from different initial conditions.

Zero leader robots Being all the robots simple followers, none of them have a desired position. As a result, the position of the load converges to a value that depends on the initial condition of the system and on the presence of internal forces, but it is not controllable at will by the robots. Regarding the attitude of the load, we can observe that:

- for $f_{\text {int }}=0$, the final attitude of the object is not uniquely determined, but it remains equal to its initial value;

- for $f_{\text {int }}>0$, the attitude of the object always converges to the desired equilibrium value $\boldsymbol{\eta}_{0}$. Therefore, $\boldsymbol{\eta}_{0}$ is an asymptotically stable attitude; 

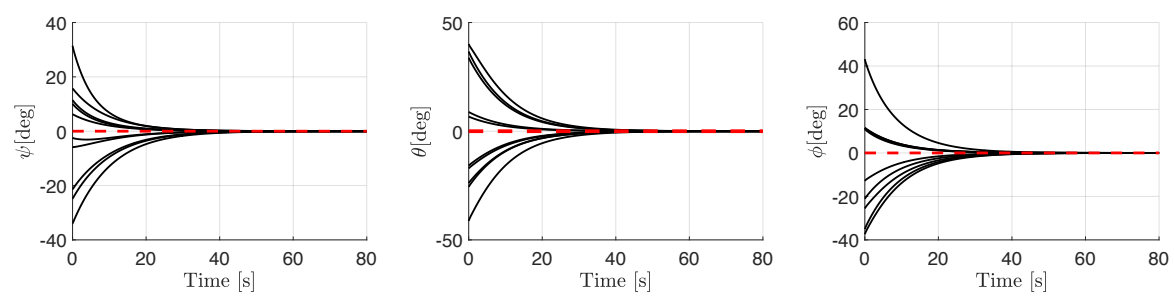

Fig. 2: Evolution of the attitude of the object with zero leader robots in a group of floating robots and $f_{\text {int }}>0$.

- for $f_{\text {int }}<0$, the attitude of the object always converges to $\boldsymbol{\eta}_{\pi}=(0,0, \pm \pi)$. Then, for $f_{\text {int }}<0$, the desired equilibrium attitude $\boldsymbol{\eta}_{0}$ is unstable while $\boldsymbol{\eta}_{\pi}$ is instead asymptotically stable.

Note that for a floating system subject to gravity, a leader-less configuration would result not applicable. Indeed, even the slightest error in estimating the mass of the object would result in the falling of the entire setup under the action of gravity. Nevertheless, this case has been included in the analysis for the sake of completeness. The results of the simulation for a floating system with $f_{\text {int }}>0$ are shown in Fig. 2. The plots highlight the important role of the internal force to control the object attitude. In fact, not even a leader robot is needed for this purpose when the internal force is non-zero. Results of the simulations for a floating system with $f_{\text {int }}=0$ and $f_{\text {int }}<0$ are collected in Fig. 8 of the appendix. Results for a ground system with $f_{\text {int }}>0, f_{\text {int }}<0$ and $f_{\text {int }}=0$ are in Fig. 9 of the appendix.

One leader robot This is the case formally analyzed in Sec. 5. Except for the case $f_{\text {int }}<0$, the tests on the floating system are analogous to the ones performed in Gabellieri et al. (2018). Nevertheless, the main results are reported here for the sake of completeness. Tests on a ground system are also presented. The results confirm what was theoretically proven in Sec. 5:

- for $f_{\text {int }}=0$, every $\boldsymbol{x} \in \mathcal{X}\left(f_{\text {int }}, \boldsymbol{q}_{L}^{d}\right)$ is stable. The pose of the load can not be unambiguously controlled to a desired configuration, as shown in Fig. 3,

- for $f_{\text {int }}>0, \boldsymbol{x}^{\prime}\left(f_{\text {int }}, \boldsymbol{q}_{L}^{d}\right)$ is asymptotically stable,

- for $f_{\text {int }}<0, \boldsymbol{x}^{\prime \prime}\left(f_{\text {int }}, \boldsymbol{q}_{L}^{d}\right)$ is asymptotically stable.

As before, the previous conclusions can be extended to a ground swarm for what concerns the position of the object and its $\psi$ angle. Figure 10 of the appendix contains the plots of the attitude for a floating system with one leader robot and $f_{\text {int }}=0$. Figures 11 and 12 of the appendix contain the plots of the attitude and of the position error for $f_{\text {int }}<0$ and $f_{\text {int }}>0$, respectively. The results for a ground system are contained in Fig. 13 of the appendix. The results presented so far can be summarized in the following observation.

Observation 1 Considering the presented communication-less approach for the manipulation of an object by a swarm of generic robots, from the theoretical previous analysis and observations from numerical simulations, we can conclude that:

1. The load orientation can be controlled to $\boldsymbol{\eta}_{0}$ even if there are no leader robots, as long as $f_{\text {int }}>0$, namely if the robots stretch the load producing an internal 

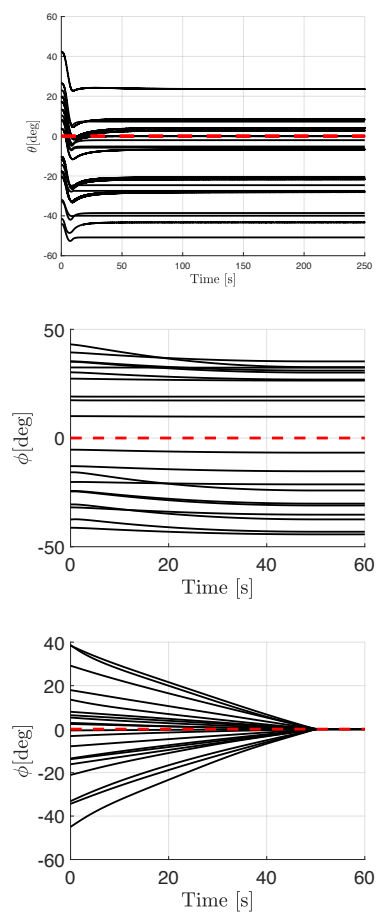
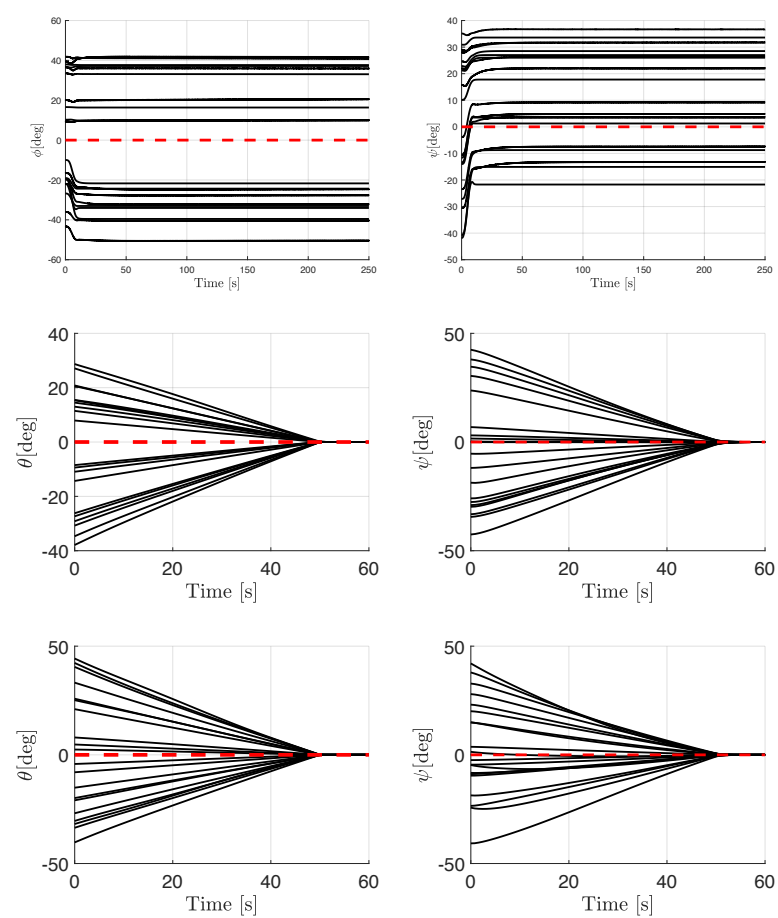

Fig. 3: Evolution of the attitude of the object in a group of floating robots with $f_{\text {int }}=0$. One leader in the first row, two leaders in the second row, and three leaders in the third row.

tension. The load orientation can still be controlled to a precise unique value if $f_{\text {int }}<0$. However, this orientation is rotated by $180^{\circ}$. The internal forces applied by the swarm to the object are sufficient alone to stabilize its attitude to a precise value; no leader is required for this specific purpose.

2. The presence of at least one leader robot is necessary for the regulation of the position of the object to a specific value.

Two leader robots We considered two leader robots anchored to the load along the $x$-axis of the body frame, $\boldsymbol{x}_{L}$, and opposite to each other. Figure 4 a shows a schematic representation of such a configuration. When the two leaders are attached to the load at the same point or very close to each other, the system behavior is very similar to the one observed with just one leader. In fact, being the leader robots anchored almost to the same point, their action is basically equivalent to the one of a single leader robot. The action of the two leader robots is much more effective when they are equally spread around the object.

Running the usual set of simulations we observed that:

- for $f_{\text {int }}=0$, the desired position of the load is now attractive. In fact, no matter the initial configuration, the load position always converges to $\boldsymbol{p}_{L}^{d}$. Regarding the attitude, the $\theta$ and $\psi$ converge always to zero. In other words, the desired attitude about the axes $\boldsymbol{y}_{L}$ and $\boldsymbol{z}_{L}$ is stable and attractive. This is not true for the attitude about $\boldsymbol{x}_{L}$, expressed by $\phi$, which is still stable but not attractive. 


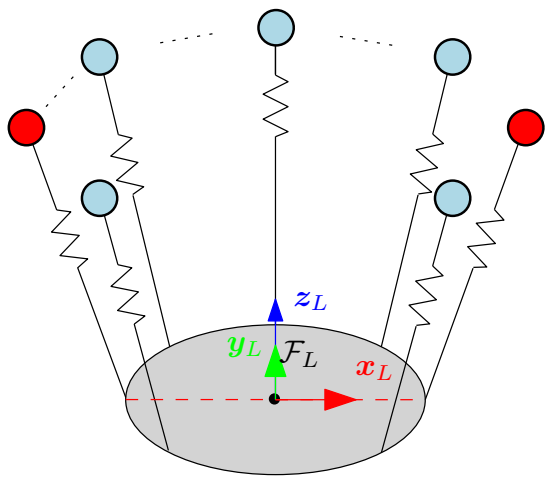

(a) Two leader robots

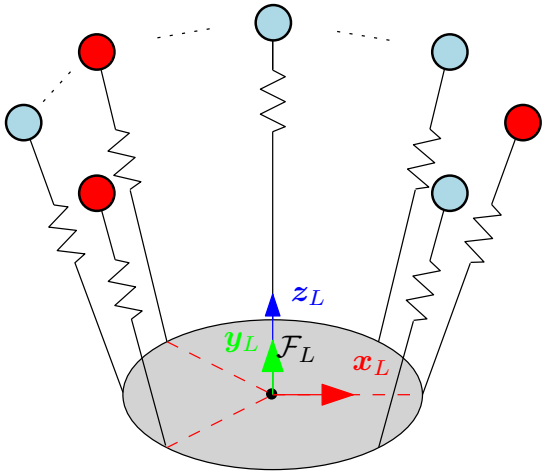

(b) Three leader robots

Fig. 4: Equally spread multiple leader robots. Each leader robot is reported as a red circle, and each follower robot as a light blue one.

Its final value is not unique and depends upon the initial configuration, as shown in Fig. 3,

- for $f_{\text {int }}>0, \boldsymbol{x}^{\prime}\left(f_{\text {int }}, \boldsymbol{q}_{L}^{d}\right)$ is asymptotically stable,

- for $f_{\text {int }}<0, \boldsymbol{p}_{L}^{d}$ converges to the desired value. Regarding the attitude, the $\psi$ and $\theta$ angles always converge to zero. On the other hand, the $\phi$ angle always converges to $\pm 180^{\circ}$, meaning that all the system flips around $\boldsymbol{x}_{L}$. This means that, for $f_{\text {int }}<0, \phi=0$ is an unstable value while $\phi= \pm 180^{\circ}$ is stable and attractive.

The results regarding the position errors and the attitude of the object for a floating system with $f_{\text {int }}=0, f_{\text {int }}>0$, and $f_{\text {int }}<0$ are contained in Fig. 14 and 15 of the appendix, respectively. Analogous results for a ground system can be found in Fig. 16 of the appendix.

Observation 2 Considering the presented communication-less approach for the manipulation of an object by a swarm of generic robots, from the previous analysis and observations, we can conclude that:

1. Two properly distributed leader robots are enough, even without any internal force, for controlling the position of the object to a unique desired value.

2. The attitude about the axes perpendicular to the line ${ }^{L} \boldsymbol{b}_{i}-{ }^{L} \boldsymbol{b}_{j}$, where $i, j$ are leaders, is always stable, no matter the value of $f_{\text {int }}$, and it converges to the desired value. On the other hand, the attitude about the axes ${ }^{L} \boldsymbol{b}_{i}-{ }^{L} \boldsymbol{b}_{j}$ converges to the desired value only if $f_{\text {int }}>0$.

3. For a ground system, two leader robots are sufficient, even without any internal force, for controlling to a desired value not only the position but also the attitude of the object, solely represented by its yaw angle.

Three leader robots As already discussed for the case when the number of leader robots is two, the most interesting scenario to examine is the one in which the leaders are evenly spread around the object. More specifically, we shall consider in these sets of simulations the distribution of leader robots schematically represented 
Table 1: Summary of the load convergence analysis.

\begin{tabular}{|c|c|c|c|}
\hline Leaders & $f_{\text {int }}$ & Position & Orientation \\
\hline 0 & $\begin{array}{l}=0 \\
>0 \\
<0\end{array}$ & $\begin{array}{l}\text { The final value depends upon } \\
\text { the initial conditions. }\end{array}$ & $\begin{array}{l}\text { The final value depends upon } \\
\text { the initial conditions. } \\
\text { Converges to } \boldsymbol{\eta}_{0} \text {. } \\
\text { Converges to } \boldsymbol{\eta}_{\pi} \text {. }\end{array}$ \\
\hline 1 & $\begin{array}{l}=0 \\
>0 \\
<0\end{array}$ & $\begin{array}{l}\text { The final value depends upon } \\
\text { the initial conditions. } \\
\text { Converges to } \boldsymbol{p}_{L}^{d} \text {. } \\
\text { Converges to a different value } \\
\text { than } \boldsymbol{p}_{L}^{d} \text {. }\end{array}$ & $\begin{array}{l}\text { The final value depends upon } \\
\text { the initial conditions. } \\
\text { Converges to } \eta_{0} \text {. } \\
\text { Converges to } \eta_{\pi} \text {. }\end{array}$ \\
\hline 2 & $\begin{array}{l}=0 \\
>0 \\
<0\end{array}$ & Converges to $\boldsymbol{p}_{L}^{d}$. & $\begin{array}{l}\text { Roll depends on the initial } \\
\text { conditions. Yaw and pitch } \\
\text { converge to the ones of } \boldsymbol{\eta}_{0} \text {. } \\
\text { Converges to } \boldsymbol{\eta}_{0} \text {. } \\
\text { Roll converges to } \pm 180^{\circ} \text {. }\end{array}$ \\
\hline 3 & $\begin{array}{l}=0 \\
>0 \\
<0\end{array}$ & Converges to $\boldsymbol{p}_{L}^{d}$. & Converges to $\boldsymbol{\eta}_{0}$. \\
\hline
\end{tabular}

in Fig. 4b. Running the usual set of simulations we observed that $\boldsymbol{x}^{\prime}\left(f_{\text {int }}, \boldsymbol{q}_{L}^{d}\right)$ is asymptotically stable independently from the value of $f_{\text {int }}$. The results regarding the position errors and the attitude of the object for a floating system with $f_{\text {int }}=0$, $f_{\text {int }}>0$, and $f_{\text {int }}<0$ are contained in Fig. 17 and 18 of the appendix, respectively. We would like to point out that for the ground case we had already obtained the same result with only two leader robots in the swarm.

Observation 3 Considering the presented communication-less approach for the manipulation of an object by a swarm of generic robots, from the previous analysis and observations, we can conclude that:

1. With three leader robots evenly distributed in the swarm, the attitude and position of the object suspended by a flying swarm converge to the desired values, regardless of the presence or the sign of the internal force.

2. For a ground swarm, having more than two evenly distributed leaders does not change the convergence behavior of the load attitude and position.

3. In both the floating and the ground cases the presence of non-zero internal forces speeds up the convergence compared to the case of zero internal forces.

In Fig. 3 one can appreciate the evolution of the attitude of a floating system with one, two and three spread leader robots when $f_{\text {int }}=0$. Figure 3 highlights the role of the presence of multiple leader robots for the attitude convergence.

The conclusions drawn until now from the numerical results presented in this section are summarized in Table 1.

\subsection{Robustness analysis}

In this section, we want to test the capability of the swarm to maintain the orientation and position of the object as close as possible to a desired value when 
external disturbances act on the object. In particular, the focus is on discovering which conditions, in terms of internal forces and number of leaders, allow the system to manipulate the object without being strongly affected by external disturbances. Constant external forces acting on the object can be compensated adding in the control law an integral action w.r.t. the load position error, as in (24) of the appendix. The relative results are shown in Fig. 19 of the appendix. Notice that the leader robot needs to measure the object position $\boldsymbol{p}_{L}$ in order to implement this modified control law. This is different w.r.t. (4) where only the information of the desired object pose was required. An integral feedback action, together with being widely used in classical robotic control, has been hypothesized in several homeostatic and adaptive processes of biological structures, from bacteria to higher organisms, see, e.g., Somvanshi et al. (2015).

On the other hand, a control action performed just by one robot is not enough to maintain the orientation of the object under external disturbance torques. This seems reasonable since each robot can only apply contact forces and not contact torques. It is therefore clear that, to react to an external torque and remain close to the desired attitude, at least a pair of contact forces are needed. In other words, a cooperative action is required for this purpose. Because of the required coordination among the robots, we think that the problem of regulating the object attitude subject to external torques is of higher interest in this context. Thus, we focus the following investigation on the attitude regulation problem. The standard control law in (4) is applied so that no robot needs to know the state of the object.

The system is always initialized at the desired equilibrium equal to $\boldsymbol{p}_{L}^{d}=$ $(0,0,0), \boldsymbol{\eta}_{0}=(0,0,0)$. In every simulation scenario, we tested the system subject to three constant external torques expressed in body frame:

1. $\boldsymbol{\tau}_{\boldsymbol{e}, \boldsymbol{x}}=m_{\text {ext }} \boldsymbol{e}_{1}$,

2. $\boldsymbol{\tau}_{\boldsymbol{e}, \boldsymbol{y}}=m_{\text {ext }} \boldsymbol{e}_{2}$,

3. $\boldsymbol{\tau}_{\boldsymbol{e}, \boldsymbol{z}}=m_{\text {ext }} \boldsymbol{e}_{3}$.

We can not expect the error to converge to zero in the presence of constant disturbances since there is no integral action. However, we wish to obtain a bounded and as small as possible steady-state error. In the following, we want to assess the robustness in terms of the deviation of the load attitude from the desired value. We say that the object attitude (or one of its components) is robust if the error stays bounded in time when subject to an external disturbance. On the contrary, it is not robust if it diverges in time.

Zero leader robots Running the tests with zero leader robots in the swarms, we observe that:

- for $f_{\text {int }}=0$, the attitude of the object is not robust,

- for $f_{\text {int }}>0$, the attitude of the object is robust

- for $f_{\text {int }}<0$, the attitude errors are all bounded. However, being $\boldsymbol{\eta}_{0}$ not even stable in the scenario with zero leader robots, as shown in the previous section, the system flips by $180^{\circ}$ around the axis of the corresponding external torque.

The results for a ground and floating system are shown in Fig. 20 and 21 of the appendix, respectively. It is not surprising that for $f_{\text {int }}=0$, none of the components of the object attitude is robust against external torques. In fact, with no robot attracted to a precise position, the only action that may induce resistance to 

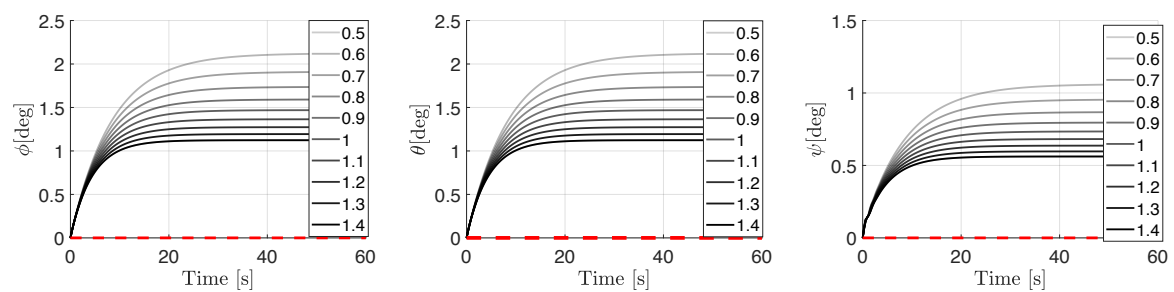

Fig. 5: Evolution of the attitude of the object with no leaders in a group of floating robots for different values of $f_{\text {int }}>0$ (displayed in the legends in N). From the left to the right, $\boldsymbol{\tau}_{\boldsymbol{e}, \boldsymbol{x}}, \boldsymbol{\tau}_{\boldsymbol{e}, \boldsymbol{y}}$, and $\tau_{e, z}$ are applied, $m_{e x t}=1 \mathrm{Nm}$. Only the rotation angle about the corresponding axis is displayed.

external disturbances is the radial stretch or compression generated by the internal forces.

In Fig. 5 we show the results for a floating swarm with no leader robots and for different positive values of $f_{\text {int }}$ keeping constant the values of $\tau_{e, x}, \tau_{e, y}, \tau_{e, z}$. We already know from the previous results that the attitude errors should stay bounded. Here, the effect of the intensity of the internal force on the steady-state error has been tested. We noticed that as soon as the intensity of the reference internal force is increased, the magnitude of the errors decreases. In particular, applying a $\tau_{\boldsymbol{e}, \boldsymbol{z}}$, the yaw angle error is smaller compared to the pitch and the roll ones, even though $m_{\text {ext }}$ has been chosen the same for each of the external torque components (see Fig. 5, last plot on the right side). This can be related to the fact that all the robots apply the same torque along $\boldsymbol{z}_{L}$ in compensating the external torque $\boldsymbol{\tau}_{\text {ext }}$. On the other hand, along $\boldsymbol{x}_{L}$, the robots whose attaching point is closer to be aligned with $\boldsymbol{x}_{L}$ apply a smaller contribution in compensating the external torque $\tau_{e, x}$. Then, the compensating action is overall less effective. Analogous results have been found for the external torque $\boldsymbol{\tau}_{\boldsymbol{e}, \boldsymbol{y}}$ along $\boldsymbol{y}_{L}$.

Observation 4 Considering the presented communication-less approach for the manipulation of an object by a swarm of generic robots, from the previous analysis and observations, we can conclude that:

1. The internal forces in the object are sufficient alone to confer robustness to the attitude of the object itself, even without any leader robot in the swarm.

2. The sensitivity of the attitude error when external disturbance torques are applied is inversely proportional to the intensity of the internal forces in the object.

One leader robot In this paragraph, we discuss the robustness to external torques when in the swarm there is one leader robot. From the simulations, we observed that:

- for $f_{\text {int }}=0$, the attitude of the object is not robust, as show in Fig. 6,

- for $f_{\text {int }}>0$, the attitude of the object is robust,

- for $f_{\text {int }}<0$, the error of each rotation angle is bounded. However, being $\boldsymbol{\eta}_{0}$ not even stable in the scenario with one leader robot and without disturbances, as shown in the previous section, the system flips about the axis of the corresponding external torque. 
The previous results are similar to the ones obtained for swarms with no leaders. The only difference is that here, when $\tau_{e, z}$ is applied, the leader robot is the pivot around which the system rotates, while in the case of no leaders, all the system rotates about the object center of mass. The results obtained in this and in the previous section enforce the conclusion that the internal forces in the object are the fundamental tool through which the swarm cooperatively controls the attitude of the object. Instead, the presence of a leader robot, despite essential for controlling the object position (as discussed in the previous section), is not crucial for controlling the attitude. Results for a ground and a floating system can be found in Fig. 22 and 23 of the appendix, respectively.

Two leader robots We shall now show the role of multiple leaders when reacting to the usual external disturbances. As in the previous section, we present here the results for two leader robots evenly distributed around the object. Specifically, we consider again the situation where the two leader robots are placed along $\boldsymbol{x}_{L}$, as in Fig. 4a.

- for $f_{\text {int }}=0$, the attitude about the axis connecting the two leader robots, $\boldsymbol{x}_{L}$, is not robust. On the other hand, the angles of rotation about the other two axes, i.e., $\boldsymbol{y}_{L}$ and $\boldsymbol{z}_{L}$, are robust, as shown in Fig. 6,

- for $f_{\text {int }}>0$, the attitude of the object is robust,

- for $f_{\text {int }}<0$, the error of each rotation angle stays bounded. However, being $\boldsymbol{\eta}_{0}$ not even stable when $\boldsymbol{\tau}_{\boldsymbol{e}, \boldsymbol{x}}$ acts on the object, the object flips of $180^{\circ}$ about $\boldsymbol{x}_{L}$, which is the axis between the two leader robots.

For the ground system all the previous results remain true, of course, the only allowed rotation, in this case, is the one about $\boldsymbol{z}_{L}$. Results for a ground and a floating system with $f_{\text {int }}=0$ and $f_{\text {int }}<0$ are shown in Fig. 25 and 24 of the appendix.

Three leader robots Let us consider here the case of three evenly distributed leader robots as in Fig. 4b. From the obtained results (for the details we refer the reader to the appendix) we can conclude that, by means of three leader robots, the control of the attitude of the object is robust, independently from the presence of the internal forces or their possible sign. The result with $f_{\text {int }}=0$ can be found in Fig. 6 . The results with $f_{\text {int }}>0$ and $f_{\text {int }}<0$ are shown in Fig. 26 of the appendix. For a ground system, this result was true with only two evenly distributed leader robots as well.

Observation 5 Considering the presented communication-less approach for the swarm manipulation of an object, from the previous results, we can conclude that:

1. Two leader robots are needed for a ground swarm to robustly control the attitude of the commonly manipulated object without exerting any internal force, namely without compressing nor stretching the object itself.

2. Three leader robots are instead necessary for the floating swarm to achieve the same objective without applying any internal force.

In Fig. 6 one can appreciate the evolution of the attitude of a floating system with one, two and three spread leader robots when $f_{\text {int }}=0$. This figure highlights the role of the presence of multiple leader robots in the attitude robustness. In 

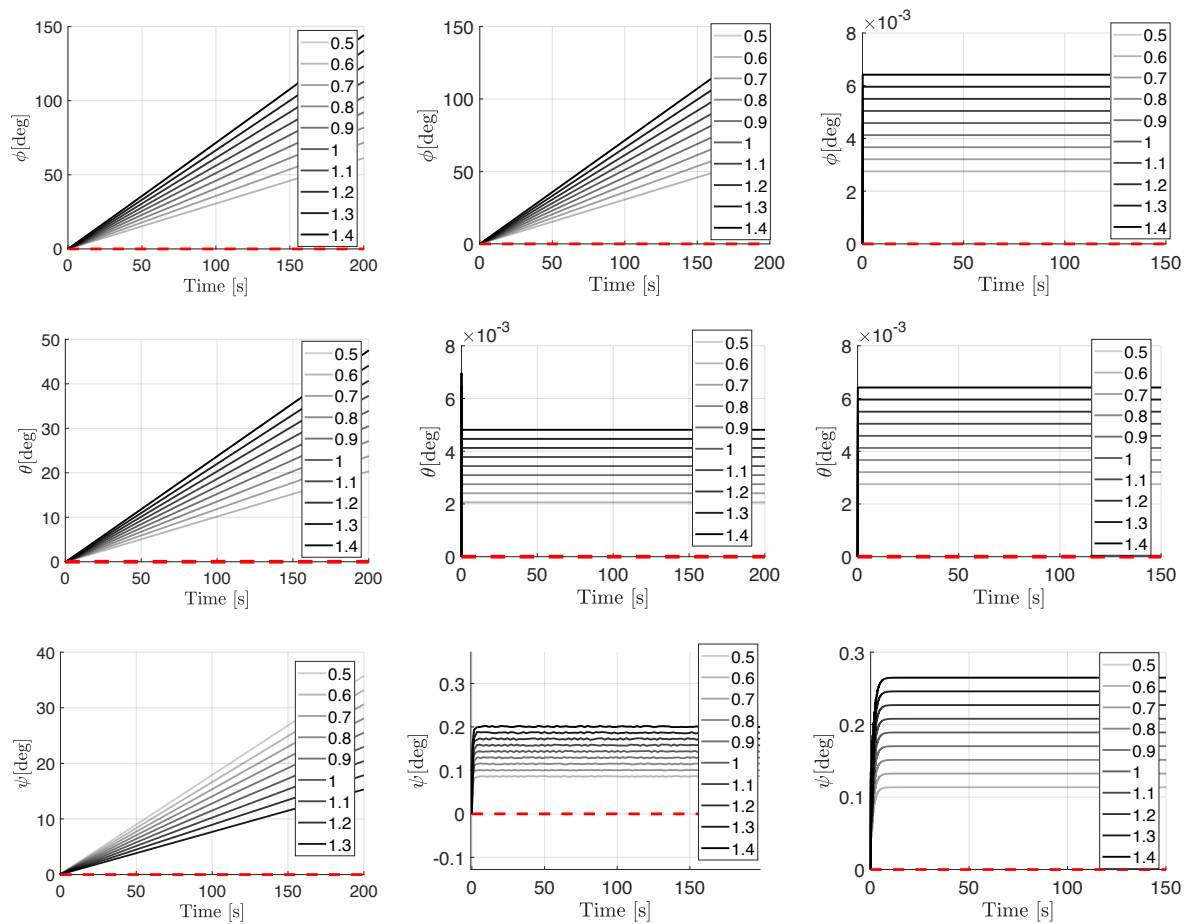

Fig. 6: Evolution of the attitude of the object in a group of floating robots with $f_{\text {int }}=0$. In each row, from the left to the right, one leader two leaders and three leaders are considered, respectively. $\tau_{e, x}$ is applied in the first row, $\tau_{e, y}$ in the second, and $\tau_{e, z}$ in the third one. Only the rotation angle about the axis corresponding to the applied torque is displayed. The legend contains the value of $m_{\text {ext }}$ expressed in $\mathrm{Nm}$.

the case of two leaders, it emerges that the roll angle $\phi$ is not robust when $\tau_{e, x}$ acts on the system, whereas the rotations about $\boldsymbol{y}_{L}$ and $\boldsymbol{z}_{L}$, i.e., the pitch and yaw angles, respectively, are robust when an external torque is applied about the corresponding axes. In the case of one leader robot, on the other hand, none of the components of the attitude are robust. This shows that the addition of one more leader robot enhances the robustness of the system when no internal forces are applied. In particular, the orientation of the load about the axes perpendicular to the axis connecting the anchoring points of the two leaders becomes robust to the effect of a disturbance of external torque. The conclusions drawn until now from the numerical results presented in this section are summarized in Table 2.

\subsection{Robustness analysis under noisy measurements}

In this subsection, the aim is to address the robustness of the attitude of the object in a more realistic scenario. We consider the external torque disturbance as random variables with a Gaussian distribution acting along all the axes of $\mathcal{F}_{L}$. We test the system under increasing mean value and with a standard deviation of $0.1 \mathrm{Nm}$. For what concerns the robots, their state and contact force measurements 
Table 2: Summary of the load robustness analysis.

\begin{tabular}{cll}
\hline Leaders & $f_{\text {int }}$ & \\
\hline & $=0$ & not robust \\
0 & $>0$ & robust \\
& $<0$ & unstable. Although, all the angles stay bounded \\
\hline & $=0$ & not robust \\
1 & $>0$ & robust \\
& $<0$ & $\eta_{0}$ is unstable. Although, all the angles stay bounded \\
\hline & $=0$ & robust, except about $x_{L}$. \\
2 & $>0$ & robust \\
& $<0$ & The desired roll angle is unstable. Nevertheless, all the angles stay \\
3 & $>0$ & robust \\
& $<0$ & robust \\
& & robust \\
\hline
\end{tabular}

are affected by noise. In particular, the estimation related to each robot, i.e., own positions and velocities, and the contact force are affected by unbiased Gaussian noise with a standard deviation of $0.01 \mathrm{~m}$ and $0.01 \mathrm{~m} / \mathrm{s}$, and $0.2 \mathrm{~N}$ respectively. The results of the simulations available in Fig. 27, 28 and 29 of the appendix for the case of one, two and three leader robots, respectively, show the qualitative convergence and robustness behavior emerged in the previous section. In Table, 3 the reader can find the mean value and the maximum standard deviation (std) of the attitude errors in the different simulated conditions (different number of leaders and different values of $f_{\text {int }}$ ). The case of zero leaders has not been included in the table results since it is not of practical relevance for the reasons previously explained. Then, the characteristics of the errors when $f_{\text {int }}>0$ are reported as well, even if three leaders are enough to have bounded attitude errors with no internal forces. Focusing on the results, first of all, it emerges that, with three leaders, the potential benefits induced by a non-zero internal force on the attitude robustness are not significant. Moreover, they also suggest that employing a group of robots with two leaders is more convenient in terms of robustness than using only one leader and that the benefits of using three leaders instead of two are not so much evident if a non-zero internal force in the object is also required.

In addition, we carried out an extensive set of simulations for the ground system under the previously described Gaussian external disturbance and noisy measurements. A viscous friction force on the object, inversely proportional to the velocity has been also considered with a dissipation coefficient equal to $0.01 \mathrm{~kg} / \mathrm{s}$ for the linear component and equal to $0.01 \frac{\mathrm{kg} \mathrm{m}^{2}}{\mathrm{~s}^{2}}$ for the rotational component. Furthermore, a static friction action has been taken into account in such a way that a force of at least $0.5 \mathrm{~N}$ is required to start the object motion. Figure 7 contains the evolution of the attitude of the ground system with zero, one and two spread leader robots for $f_{\text {int }}=0, f_{\text {int }}>0, f_{\text {int }}<0$. Then, still in Fig. 7 , for each case, four simulations, corresponding to increasing mean values of the disturbance torque, are presented (for a total of 36 simulations). The mean value and the maximum standard deviation of the attitude error for a ground system are instead in Table 4. 
Table 3: Summary of the statistics of the attitude errors for a floating system.

\begin{tabular}{cccc}
\hline Leaders & $f_{\text {int }}$ & error mean [deg] & error maximum std [deg] \\
\hline \multirow{3}{*}{1} & \multirow{3}{*}{$f_{\text {int }}>0$} & $\psi:-6.2867$ & $\psi: 2.6101$ \\
& & $\theta:-33.6865$ & $\theta: 4.1459$ \\
& $\phi: 25.4101$ & $\phi: 3.8239$ \\
\hline \multirow{3}{*}{2} & \multirow{2}{*}{$f_{\text {int }}>0$} & $\psi: 0.5273$ & $\psi: 0.3659$ \\
& & $\theta: 0.0129$ & $\theta: 0.5232$ \\
& $\phi: 2.1274$ & $\phi: 0.3746$ \\
\hline \multirow{2}{*}{3} & \multirow{2}{*}{$f_{\text {int }}>0$} & $\psi: 0.0863$ & $\psi: 0.2477$ \\
& & $\phi:-0.0380$ & $\theta: 0.7354$ \\
& & $\phi: 0.1902$ & $\phi: 0.7820$ \\
\hline \multirow{2}{*}{3} & \multirow{2}{*}{$f_{\text {int }}=0$} & $\theta: 0.0552$ & $\psi: 0.2526$ \\
& & $\phi: 0.0875$ & $\theta: 0.1726$ \\
& & & $\phi: 0.1762$ \\
\hline
\end{tabular}

Table 4: Summary of the statistics of the attitude errors for a ground system.

\begin{tabular}{cccc}
\hline Leaders & $f_{\text {int }}$ & error mean $[\mathrm{deg}]$ & error maximum std $[\mathrm{deg}]$ \\
\hline 0 & $f_{\text {int }}>0$ & 1.9475 & 2.4201 \\
\hline 1 & $f_{\text {int }}>0$ & 2.4513 & 3.7961 \\
\hline 2 & $f_{\text {int }}>0$ & 0.0862 & 0.3580 \\
\hline 2 & $f_{\text {int }}=0$ & 0.0674 & 0.3911 \\
\hline
\end{tabular}

We remark that, even though the behavior of the attitude is the same with zero and one leader, more advantages can be appreciated with one leader. In fact, by means of the leader, the position of the object can be controlled as well. On the contrary, with no leader robots, there is no position reference and the system is free to wander under external disturbance.

\section{Discussion and future work}

We presented a generic model and a control law for robots cooperatively manipulating an object, which can be considered valid both for ground and floating systems. The presented approach is not based on explicit communication, but rather on the force exchanged through the physical interactions. The manipulated object becomes an implicit means of information exchange. The proposed method employs a leader-follower approach where the follower robots, while damping the oscillations of the system, try to bring the sensed contact force (exchanged with the object) to a desired value. On the other hand, a leader robot, in addition to the action performed by a follower robot, tries to move, and, as a result, the global system moves toward a specific point.

We were interested at first in the capability of the swarms of bringing the cooperatively manipulated object to a specific equilibrium configuration (both position and orientation). In addition, we were interested in the capability of the swarms of keeping the position and the attitude of the object close to the desired 

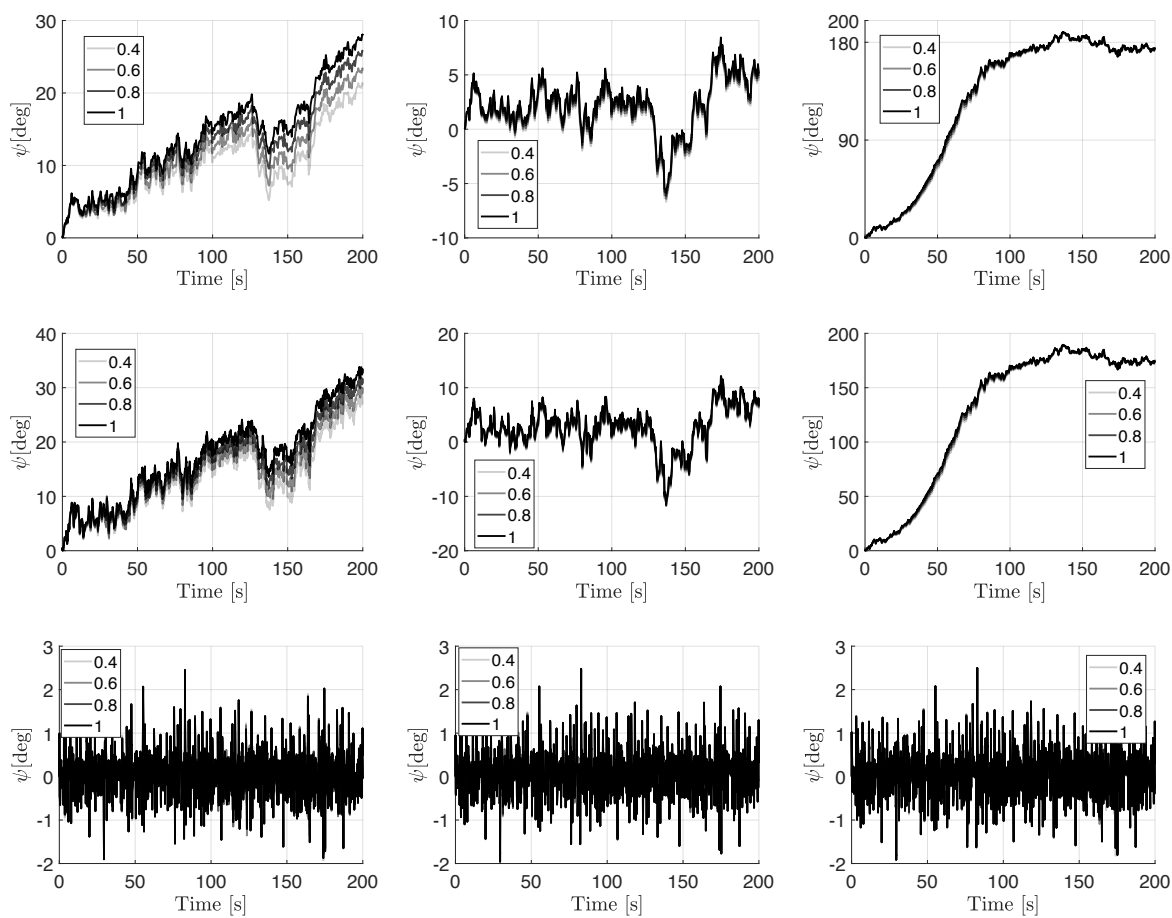

Fig. 7: Evolution of the attitude of the object in a group of ground robots. The system is subject to an external Gaussian-distributed disturbance torque applied to the object center of mass, with increasing mean value and $0.1 \mathrm{Nm}$ standard deviation. The mean value of the disturbance along the three orthogonal directions is reported in the legend in $\mathrm{Nm}$. $f_{\text {int }}=0$ in the first column, $f_{\text {int }}>0$ in the second, and $f_{\text {int }}<0$ in the third one. In the first, second and third row are the results for zero, one and two spread leader robots, respectively.

values when external disturbances are applied. This is in our opinion an important aspect to study in order to model more realistic conditions.

With those goals, we formally studied the equilibria of the system with one leader robot and characterized their stability according to the value of the required internal force in the object. From the results coming from our analytical analysis, we can argue that the desired equilibrium configuration is stable, but not attractive if no reference internal force is set. The desired equilibrium configuration is asymptotically stable or unstable if a positive or negative reference internal force is set, respectively. Through extensive numerical simulations, we confirmed these results and we drew additional ones about the stability and the robustness of the system against external disturbances. The numerical study has been conducted varying the value of the reference internal force in the object as well as the number of leader robots.

More specifically, through the numerical analysis, we showed that an internalforce reference is the fundamental tool through which the swarm regulates the attitude of the object. For this purpose, no leader robot is indeed required. The presence of at least one leader robot is instead crucial for controlling the object position to a desired value. We also found that two evenly distributed leader robots 
are sufficient for a ground swarm to control both the position and the orientation of the object to a uniquely determined value, not generating any compression nor tension in the object. To accomplish the same control objective in a floating swarm, the presence of at least three leader robots is required.

Furthermore, the results highlighted the fundamental role of the internal forces also in the robustness of the attitude control, namely in maintaining bounded attitude errors when external disturbances are applied to the system. The presence of one leader does not change the robustness properties compared to the case with no leaders. Actually, to have the robustness of at least some components of the object attitude with a zero internal force reference, at least two leader robots spread in the group are required. Two leader robots evenly distributed in the group are indeed enough for a ground swarm to robustly control the object without setting any reference internal forces, while three leader robots are necessary in a floating swarm. In the case of only two leaders in a floating swarm, the rotation about the axis connecting the two leader robots is not robust if no internal forces are applied to the object. We can finally state that one leader robot and positive reference internal forces are enough to robustly control the position and the attitude of a ground or floating object.

We conducted a thorough formal and numerical analysis to draw our conclusions, but of course, the implementation of the control law on real platforms remains a very important future work to further validate the results and deepen the analysis. For the real implementation of our algorithm, a good force sensor for each robot or a force observer would be required, together with a fast position controller, as well as some effective obstacle avoidance protocols in order to avoid possible collisions among the robots.

Acknowledgements This research was partially supported by the ANR, Project ANR-17CE33-0007 MuRoPhen. 

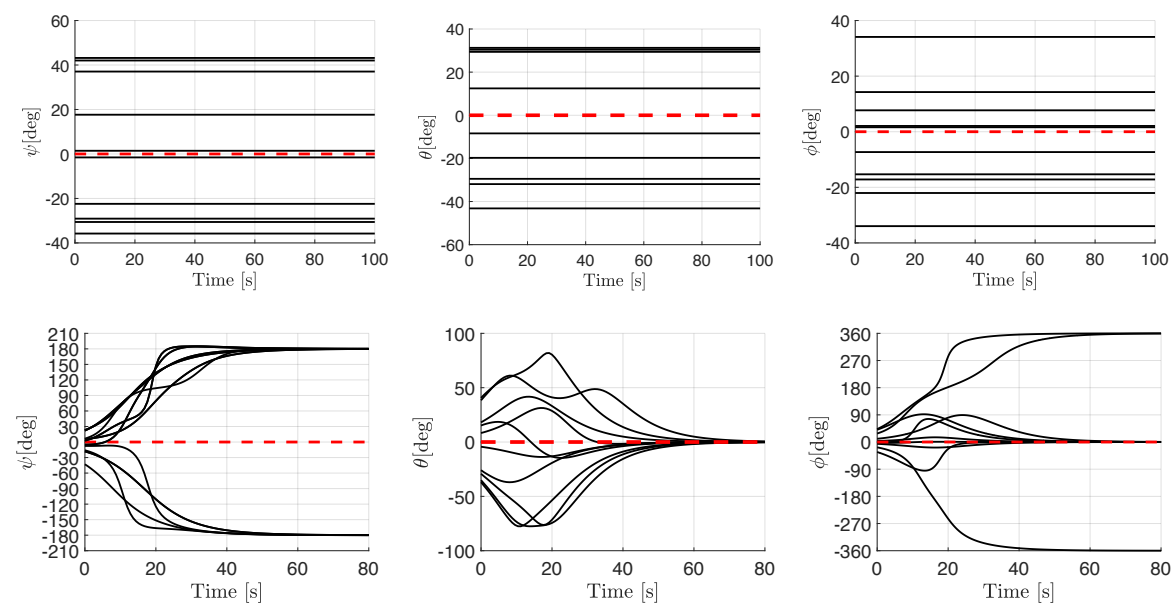

Fig. 8: Evolution of the attitude of the object with zero leader robots in a group of floating agents. $f_{\text {int }}=0$ in the first row, $f_{\text {int }}<0$ in the second row. There is convergence of the roll angle to the same value also in the last plot, in fact $\phi=0,360^{\circ},-360^{\circ}$ correspond to the same configuration.

\section{A Appendix}

\section{A.1 Introduction}

In Sec. A.2 we integrate the simulation results concerning the convergence analysis presented in Sec. 6.1 of the manuscript.

Then, in Sec. A.3 we give more details about the modified leader-robot control law (modification of equation (4) of the manuscript) that allows to achieve convergence of the object position to a desired value in presence of external disturbanceforces. Numerical validation of the effectiveness of the proposed control law is also provided. In the same section, we integrate the simulation results relative to Sec. 6.2 of the manuscript. Eventually, in Sec. A.4 we integrate the simulation results presented in Sec. 6.3 of the manuscript.

\section{A.2 Convergence analysis}

Zero leader robots Figures 8 and 9 show the results of the simulations for a floating and a ground system, respectively. From Fig. 9 one can see that the attitude of the object is unstable when $f_{\text {int }}<0$ and is identically equal to its initial value when $f_{\text {int }}=0$. The plots of the object position is not reported here since the position only depends on the initial conditions: a swarm with no leader robots has no control over the position of the object. From Fig. 9 one can see that, similarly to what is shown in the manuscript for the floating case, also in the ground case the attitude converges to the desired value when $f_{\text {int }}>0$.

One leader robot The results for a floating swarm with $f_{\text {int }}=0, f_{\text {int }}<0$, and $f_{\text {int }}>0$ are reported in Fig. 10, Fig. 11, and Fig. 12, respectively. From Fig. 10 

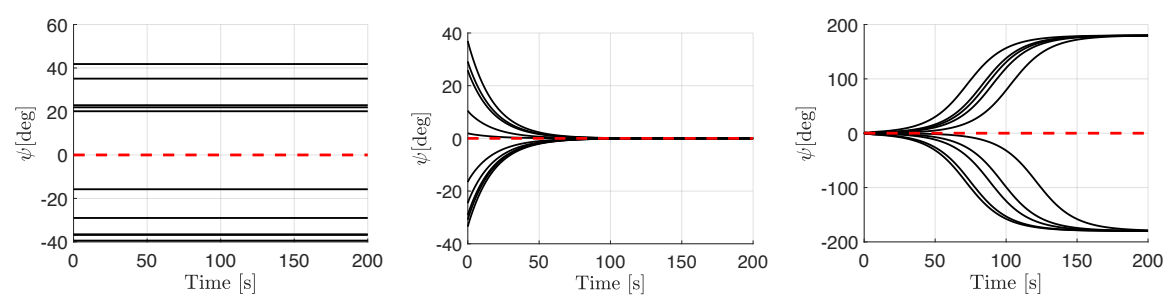

Fig. 9: Evolution of the attitude of the object (just yaw angle) with zero leader robots in a group of ground agents. From the left to the right, $f_{\text {int }}=0, f_{\text {int }}>0$, and $f_{\text {int }}<0$.
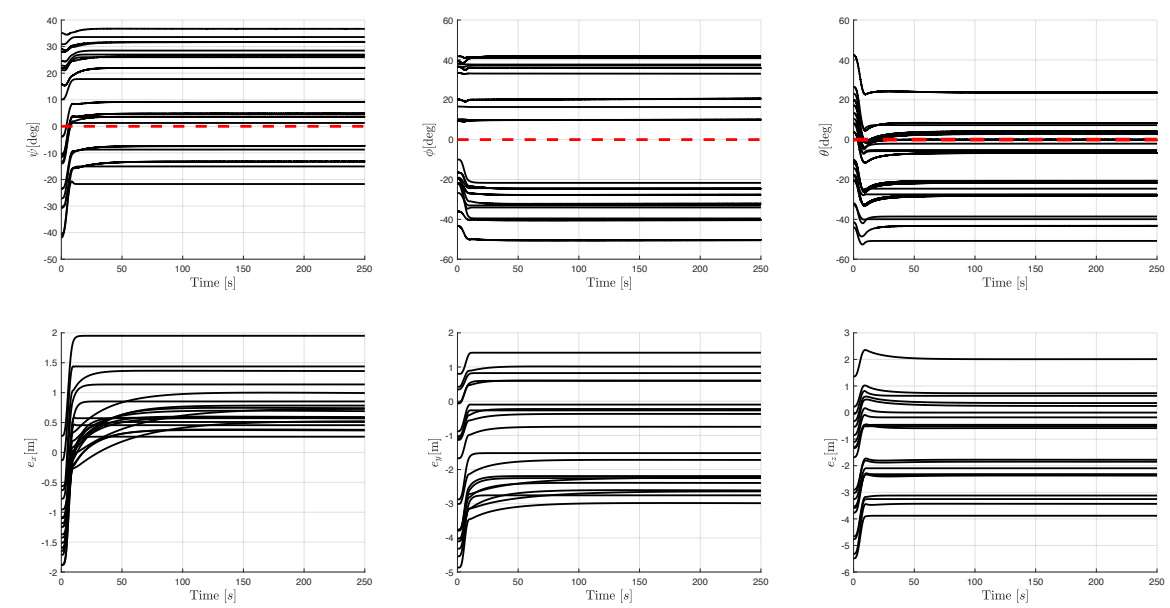

Fig. 10: Evolution of the attitude (first row) and of the position error (second row) of the object with one leader in a group of floating agents and $f_{\text {int }}=0$.

one can see that nor the position neither the attitude of the manipulated object are controllable for $f_{\text {int }}=0$, whereas from Fig. 12 it emerges that they converge to the desired values for $f_{\text {int }}>0$. Finally, from Fig. 11 one can see that for $f_{\text {int }}<0$ the position and the attitude of the object do not converge to the desired values; yet they converge to a configuration equal to the desired one but rotated by $180 \mathrm{deg}$ around the vertical axis. Additionally, the same holds for a ground swarm. The relative results can be found in Fig. 13. 

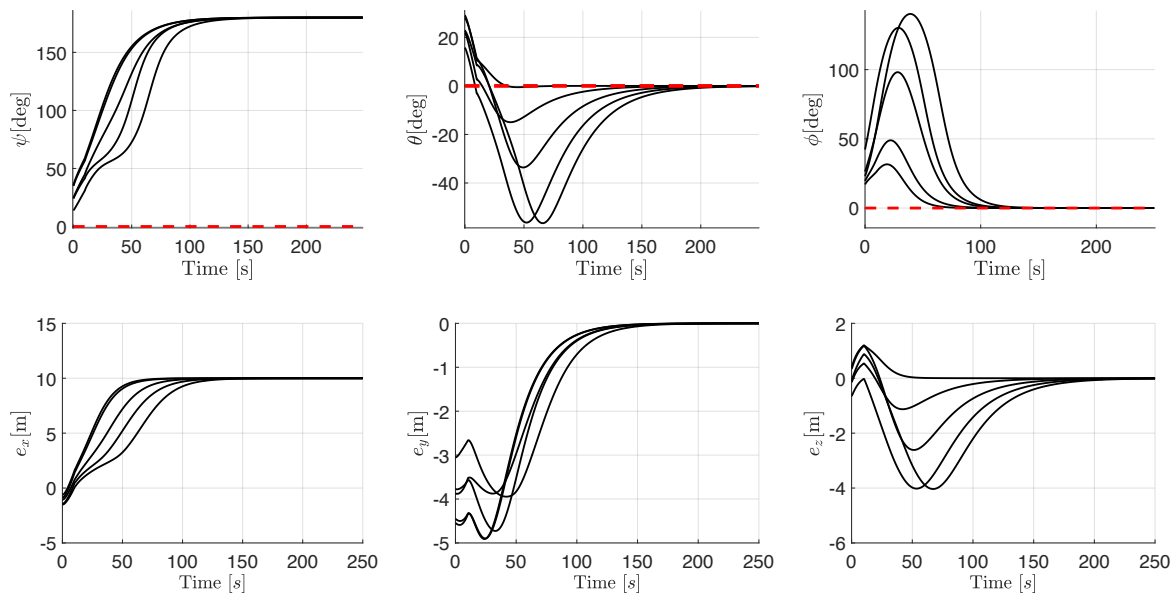

Fig. 11: Evolution of the attitude (first row) and of the position error (second row) of the object with one leader in a group of floating agents and $f_{\text {int }}<0$.
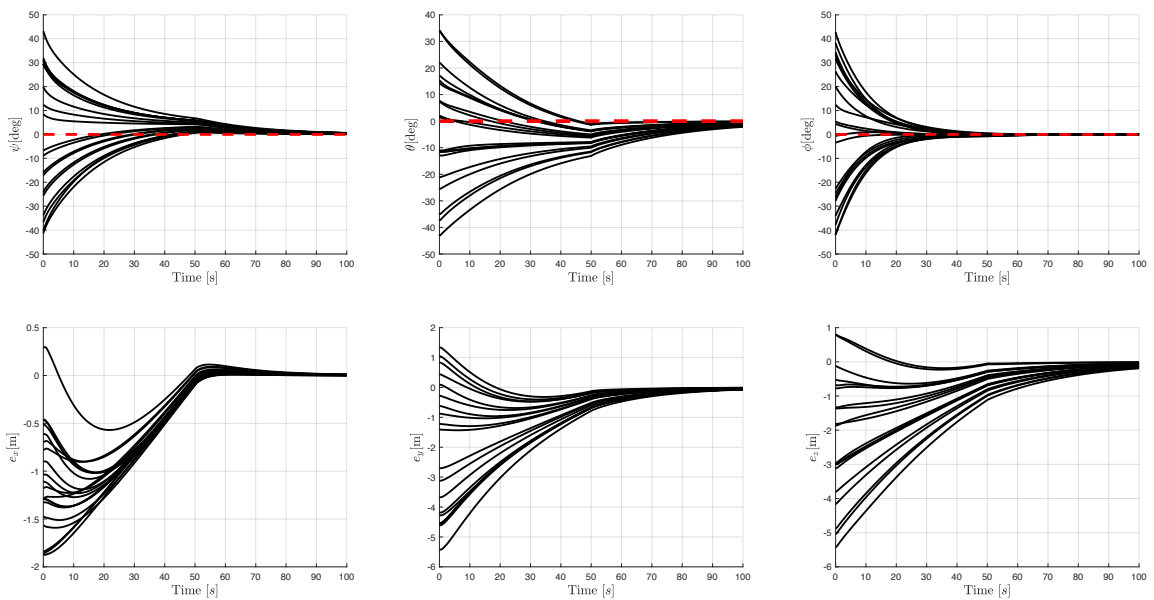

Fig. 12: Evolution of the attitude (first row) and of the position error (second row) of the object with one leader in a group of floating agents and $f_{\text {int }}>0$. 

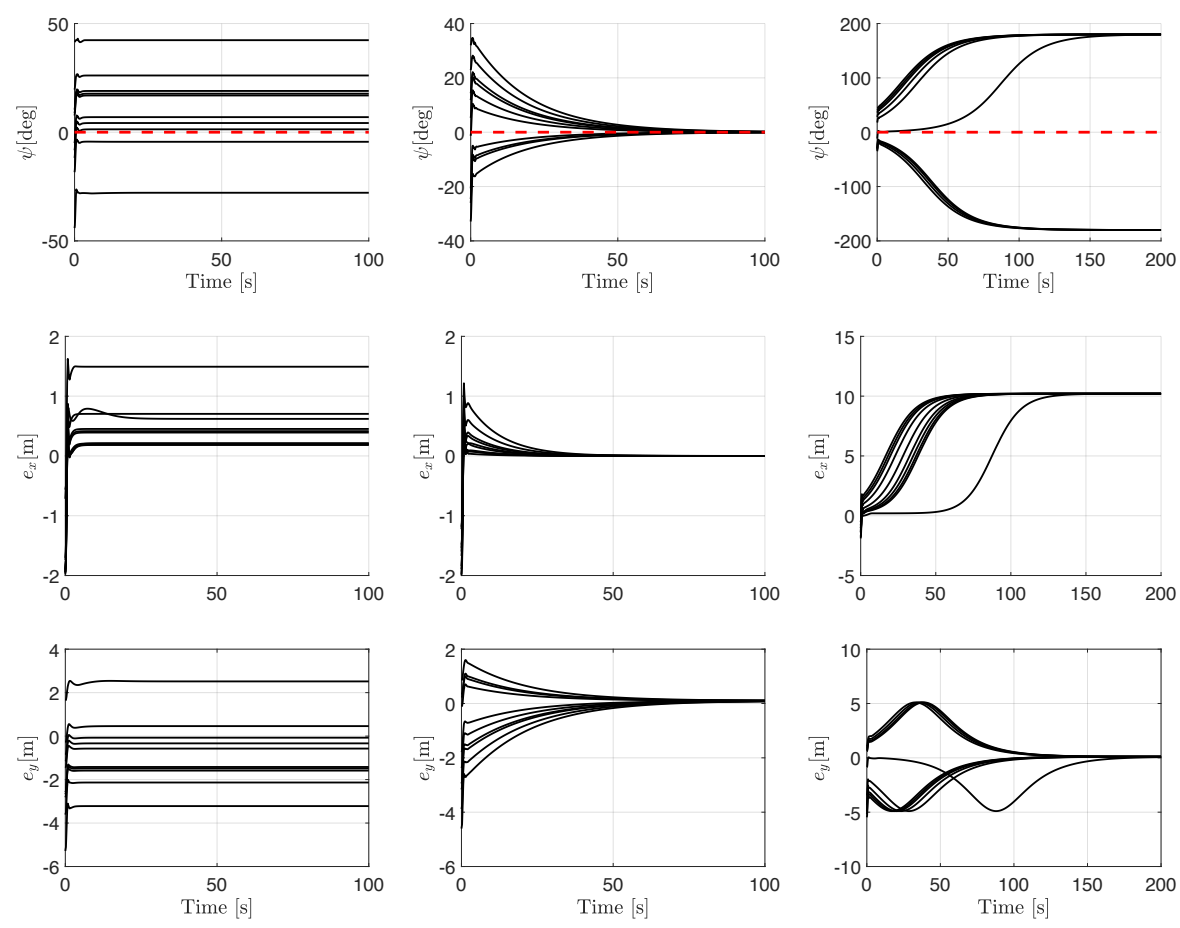

Fig. 13: Evolution of the attitude (first row) and of the position error (second row) of the object with one leader in a group of ground agents. From the left to the right, $f_{\text {int }}=0, f_{\text {int }}>0$, and $f_{\text {int }}<0$. 

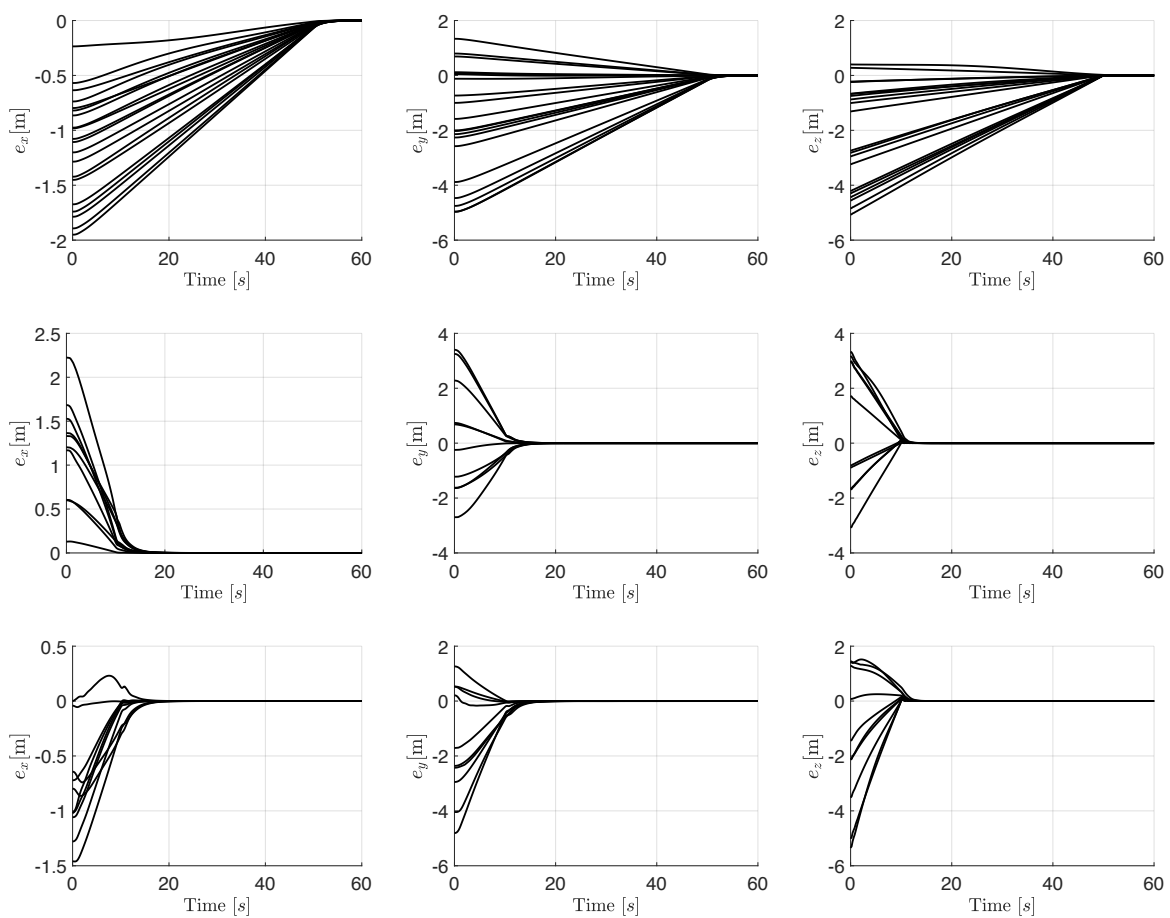

Fig. 14: Position error of the object with two leaders in a group of floating agents. $f_{\text {int }}=0$ in the first row, $f_{\text {int }}>0$ in the second row, and $f_{\text {int }}<0$ in the third row.

Two leader robots The results of the simulations relative to the position and orientation of the object in a floating system can be found in Fig. 14 and Fig. 15, respectively. We would like to stress out that the configurations of the object corresponding to $\phi= \pm 180^{\circ}$ are actually equal. In fact, what changes is the way in which the configuration is reached, namely by rotating clockwise or counterclockwise about the $\boldsymbol{x}_{L}$ axis. The results for the ground case are in Fig. 16. From Fig. 14 one can see that the position of the object is now controlled to the desired value despite the value or presence of the internal force. This is not true for the attitude of a floating system if $f_{\text {int }}=0$, as it is shown in Fig. 15. On the other hand, Fig. 16 shows that for a ground system two leader robots are enough to control both position and orientation of the object, despite the value assumed by the internal force.

Three leader robots The numerical results for the floating case are shown in Fig. 17 and Fig. 18. From those figures one can see that both the attitude and the position of the object converge to the desired values, despite the value assumed by $f_{\text {int }}$. 

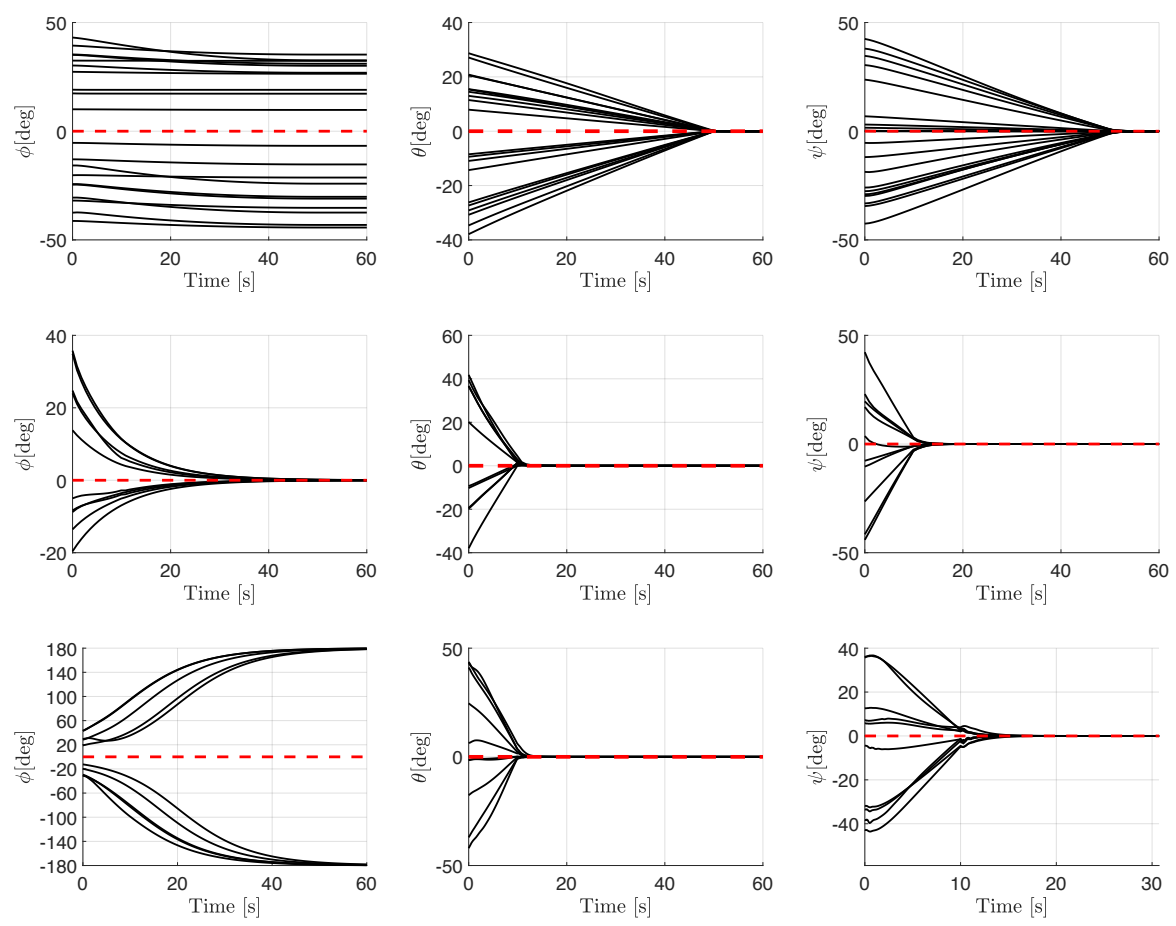

Fig. 15: Evolution of the attitude of the object with two leaders in a group of floating robots. $f_{\text {int }}=0$ in the first row, $f_{\text {int }}>0$ in the second row, and $f_{\text {int }}<0$ in the third row. 

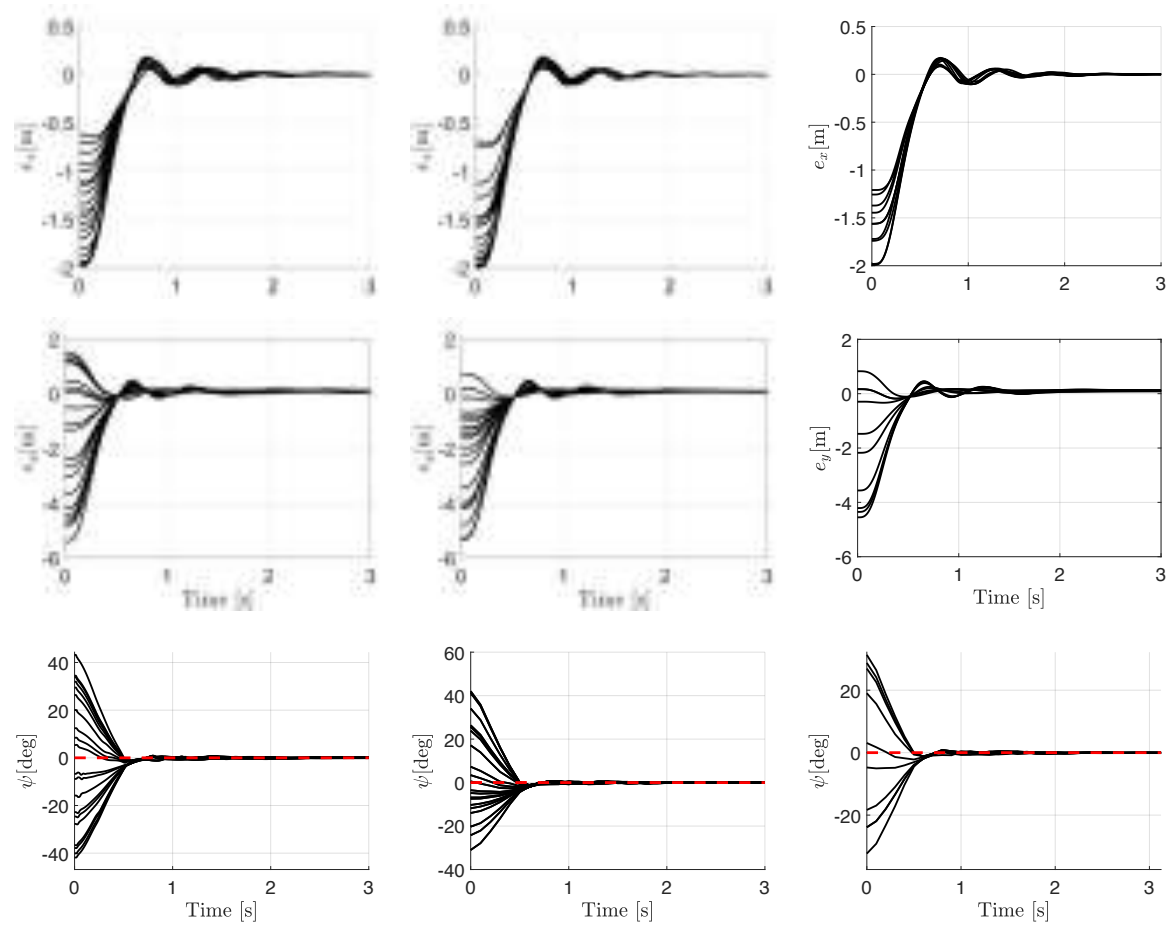

Fig. 16: Position error (first and second rows) and attitude (third row) of the object with two leaders in a group of ground agents. From the left to the right, $f_{\text {int }}=0, f_{\text {int }}>0, f_{\text {int }}<0$ 

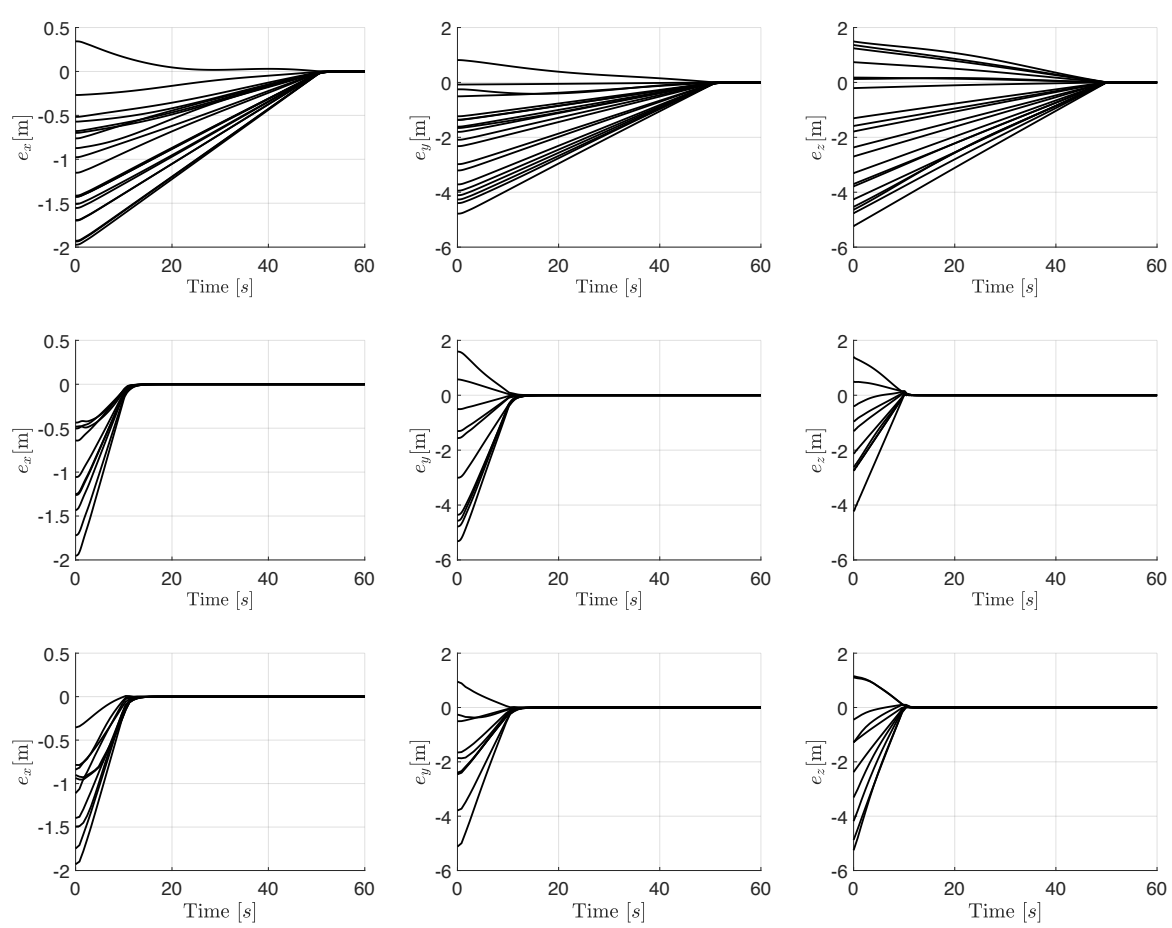

Fig. 17: Position error of the object with three leaders in a group of floating agents. From the first row to the third, $f_{\text {int }}=0, f_{\text {int }}>0, f_{\text {int }}<0$. Notice that, even though the position error converges to zero in each case, the presence of non-zero internal forces increases the convergence rate. 

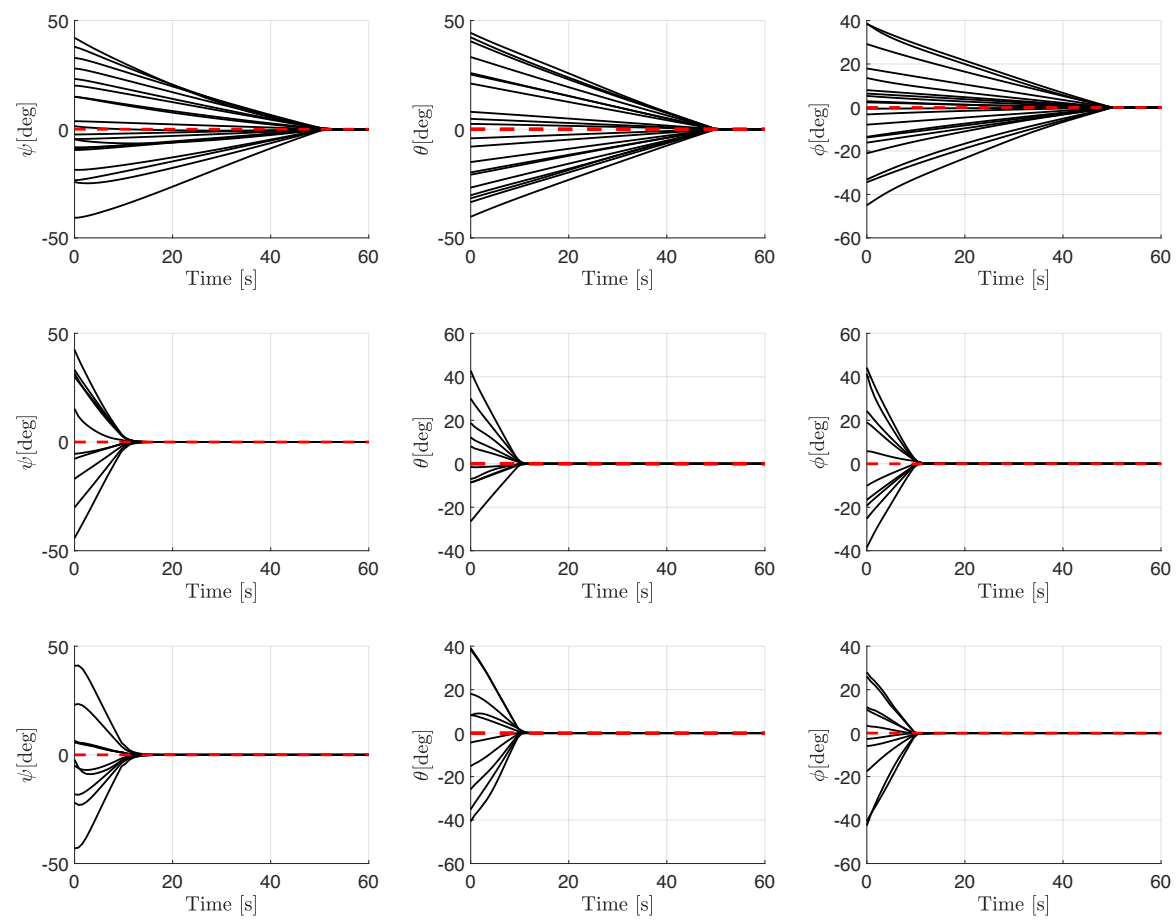

Fig. 18: Evolution of the attitude of the object with three leaders in a group of floating agents. $f_{\text {int }}=0$ in the first row, $f_{\text {int }}>0$ in the second row, and $f_{\text {int }}<0$ in the third row. Notice that, even though the attitude converges to the desired value in each case, the presence of non-zero internal forces increases the convergence rate. 

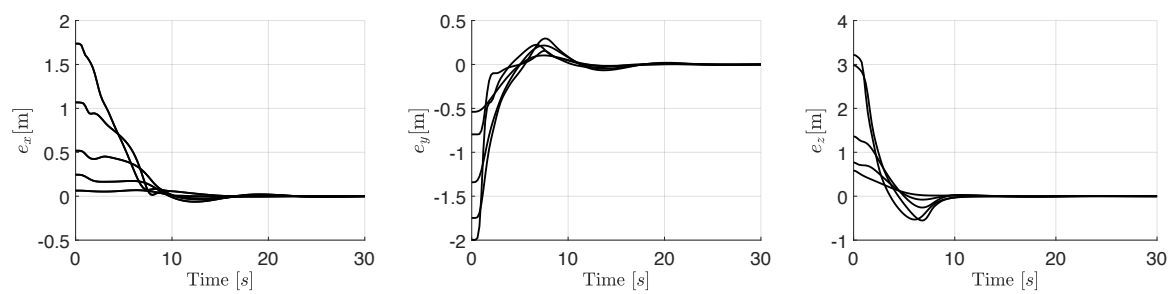

Fig. 19: Evolution of the position error in case of one leader in the swarm of floating agents and no internal forces. The leader agent applies the control force of eq. (24). An external disturbance force of $0.5 \mathrm{~N}$ along all the three directions is applied to the object center of mass. $\boldsymbol{K}_{1}^{I}=10^{5} \mathrm{~N} / \mathrm{ms}$. The system is initialized in different configurations.

\section{A.3 Robutness analysis}

The modified control law of the leader robot (modification of eq. (4) of the manuscript) that allows to robustly control the object position in presence of external forces is:

$$
\boldsymbol{f}_{C i}=-\boldsymbol{B}_{i} \dot{\boldsymbol{p}}_{R i}-\boldsymbol{K}_{i}\left(\boldsymbol{p}_{R i}-\boldsymbol{p}_{R i}^{d}\right)+{ }^{W} \boldsymbol{\pi}_{i}+\boldsymbol{K}_{i}^{I} \int\left(\boldsymbol{p}_{L}^{d}-\boldsymbol{p}_{L}\right) d t .
$$

In more detail, we added a standard integral term on the position error of the object with proper gain $\boldsymbol{K}_{i}^{I} \in \mathbb{R}^{3 \times 3}$.

In Fig. 19 we show that, with the modified control policy expressed in (24), one leader is enough to drive the position of the object to the desired value in the presence of an external disturbance force, even when the swarm applies no internal forces on the object.

In the following of this section, results concerning the robustness of the attitude of the object in presence of external torques are presented.

Zero leader robots In Fig. 20 the results for a ground system are displayed, showing that a non-zero internal force in the object is sufficient to robustly control its attitude even with zero leader robots. In Fig. 21 the two cases without internal forces and with positive internal forces in the object are displayed for the floating case. The figure shows that the presence of a non-zero internal forces allows to keep the attitude errors bounded.

One leader robots Results for a floating system are in Fig. 23, from which it emerges that the presence of $f_{\text {int }}>0$ makes the attitude robust to external disturbances. Analogous results for a ground system are displayed in Fig. 22. 

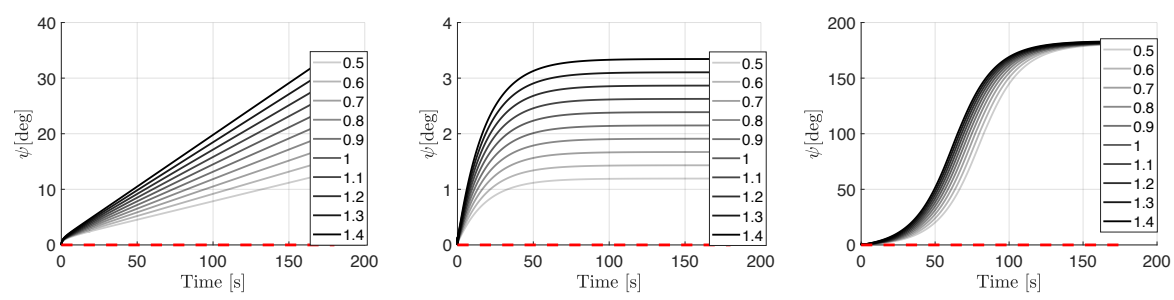

Fig. 20: Evolution of the attitude of the object with no leader in a group of ground agents. $f_{\text {int }}=0$ in the first column, $f_{\text {int }}>0$ in the second column, and $f_{\text {int }}<0$ in the third column. The external disturbance torque $\boldsymbol{\tau}_{e, z}$ is applied. We recall that its intensity $m_{e x t}$, is expressed in the legend in Nm.
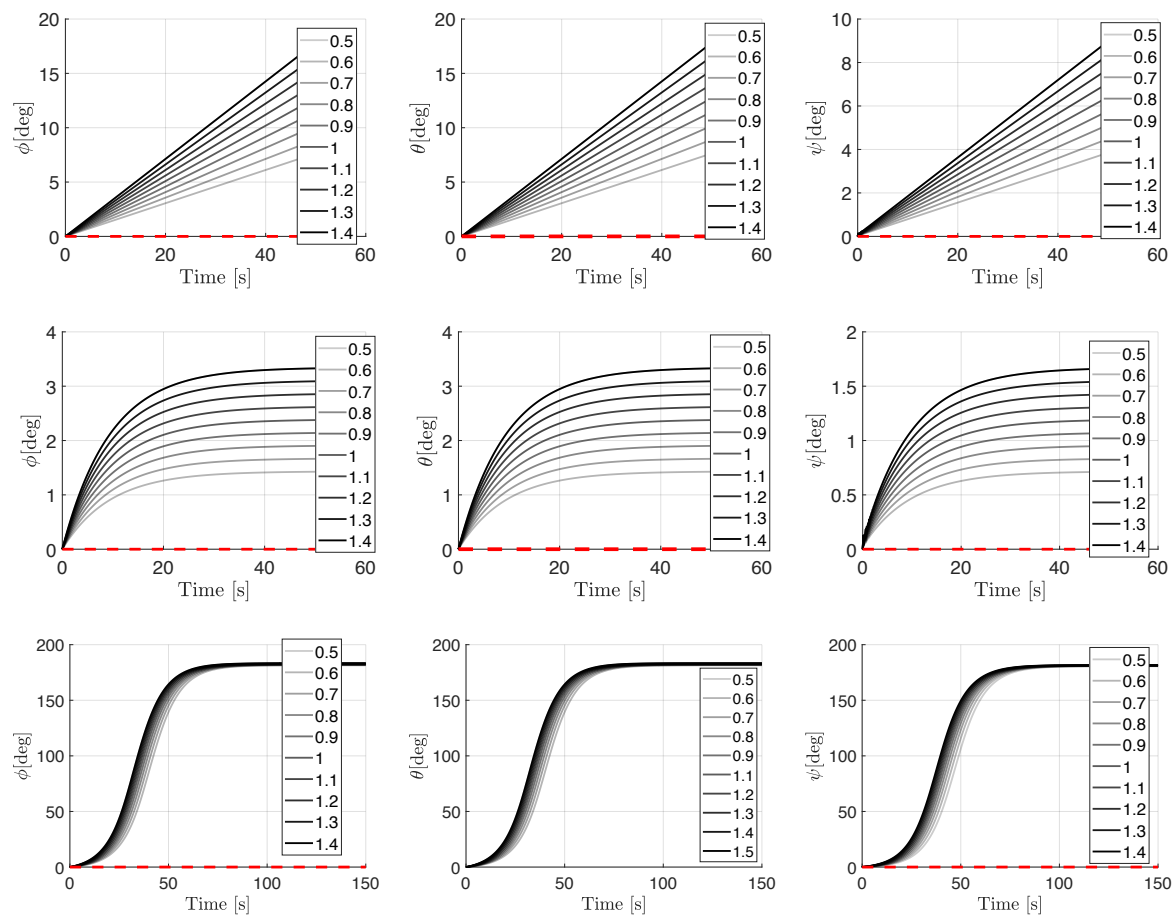

Fig. 21: Evolution of the attitude of the object, perturbed by external disturbance torques applied at its center of mass, with no leaders in a group of floating agents. $f_{\text {int }}=0$ in the first row, $f_{\text {int }}>0$ in the second row, and $f_{\text {int }}<0$ in the third row. From the left to the right, $\tau_{e, x}, \tau_{e, y}$, and $\tau_{e, z}$ are applied. Only the rotation around the corresponding axes is displayed, because it is the only one significantly varying. The legends contains the value of $m_{\text {ext }}$ expressed in $\mathrm{Nm}$. 

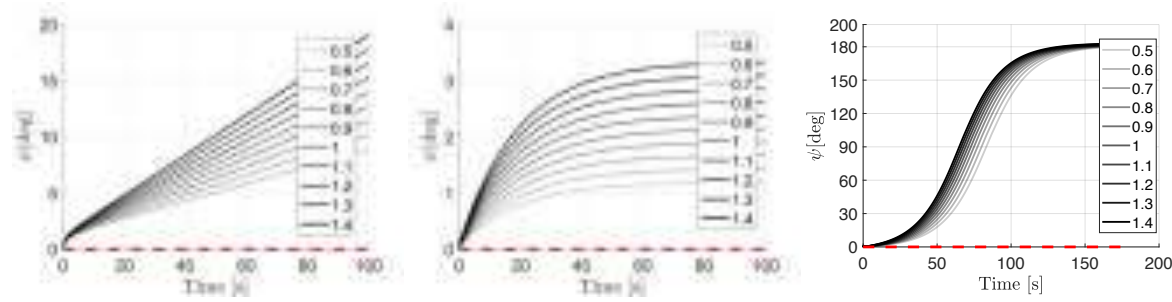

Fig. 22: Evolution of the attitude of the object with one leader in a group of ground agents. $f_{\text {int }}=0$ in the first column, $f_{\text {int }}>0$ in the second column, and $f_{\text {int }}<0$ in the third column. The external disturbance torque $\boldsymbol{\tau}_{e, z}$ is applied. We recall that its intensity $m_{e x t}$, is expressed in the legend in $\mathrm{Nm}$.
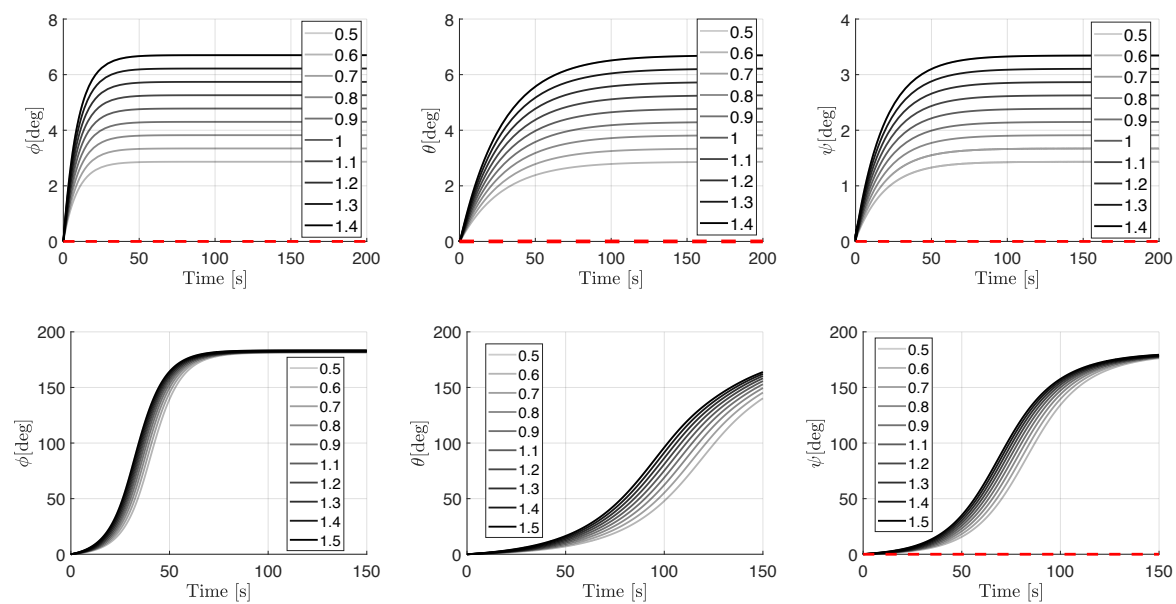

Fig. 23: Evolution of the attitude of the object with one leader in a floating swarm with $f_{\text {int }}>0$ in the first row and $f_{\text {int }}<0$ in the second row. From the left to the right, $\boldsymbol{\tau}_{\boldsymbol{e}, \boldsymbol{x}}, \boldsymbol{\tau}_{\boldsymbol{e}, \boldsymbol{y}}$, and $\boldsymbol{\tau}_{\boldsymbol{e}, \boldsymbol{z}}$ are applied, and only the rotation angle about the corresponding axis is displayed. The legends contains the value of $m_{\text {ext }}$ expressed in $\mathrm{Nm}$. 

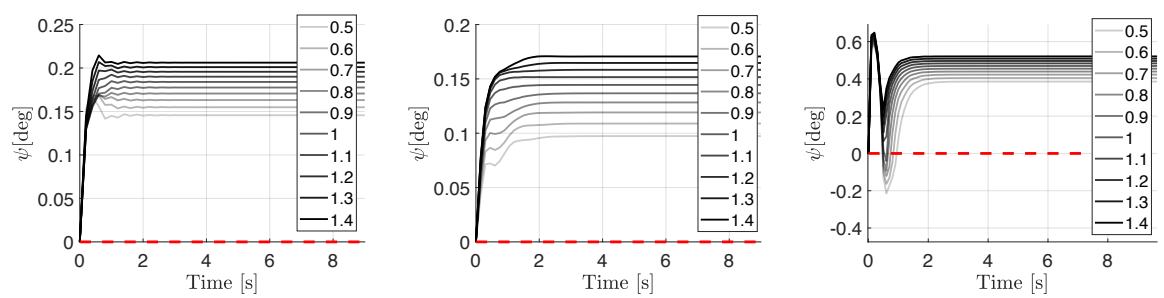

Fig. 24: Evolution of the attitude of the object with two leaders in a group of ground agents. $f_{\text {int }}=0$ in the first column, $f_{\text {int }}>0$ in the second column, and $f_{\text {int }}<0$ in the third column. The external disturbance torque $\tau_{e, z}$ is applied. We recall that its intensity is $m_{e x t}$, is expressed in the legend in $\mathrm{Nm}$.

Two leader robots Results for a ground system and for a floating one are in Fig. 24 and in Fig. 25, respectively. From Fig. 24 it emerges that two evenly dustributed leaders are sufficient to robustly control the attitude of a ground system even when $f_{\text {int }}=0$. Instead, from Fig. 25 , one can see that the presence of two evenly distributed leaders in a floating swarm confers robustness only to some components of the object attitude. To robustly control the entire attitude of the object, $f_{\text {int }}>0$ is required in a floating system. 

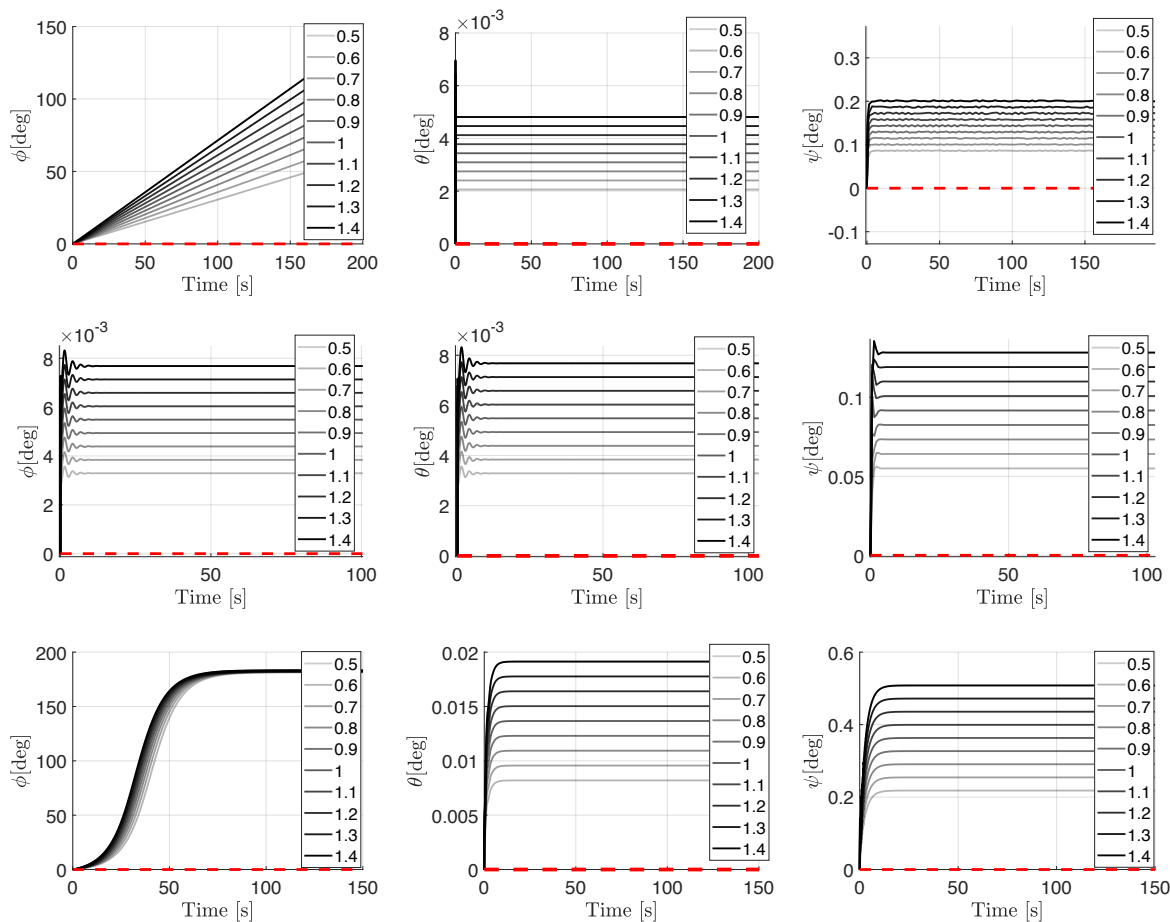

Fig. 25: Evolution of the attitude of the object with two leaders in a group of floating agents. $f_{\text {int }}=0$ in the first row, $f_{\text {int }}>0$ in the second row, and $f_{\text {int }}<0$ in the third row. From the left to the right, $\tau_{e, x}, \tau_{e, y}$, and $\tau_{e, z}$ are applied, and only the rotation angle about the corresponding axis is displayed. The legends contains the value of $m_{\text {ext }}$ expressed in $\mathrm{Nm}$. In some cases there are oscillations due to the dynamic behavior of the system, which can be tuned by changing the control parameters of the agents. 

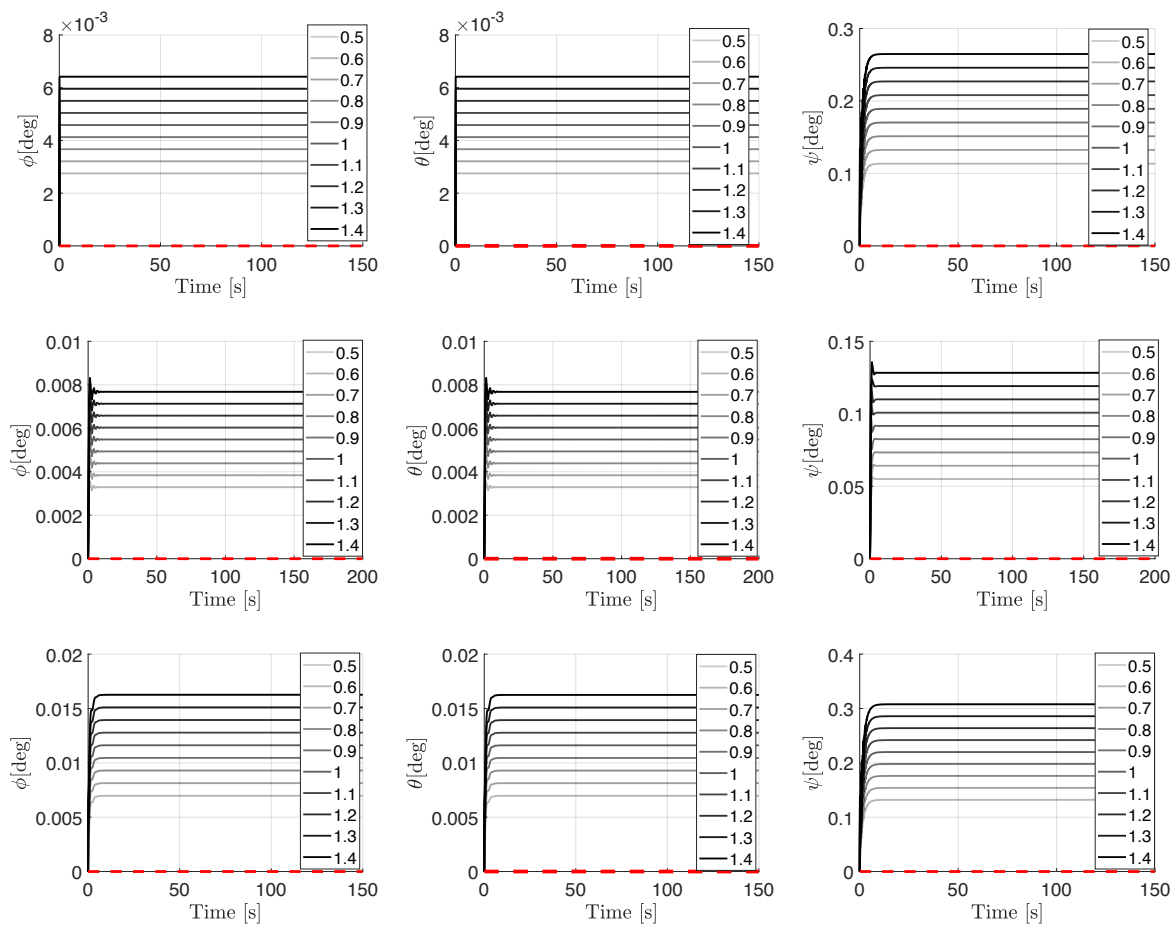

Fig. 26: Evolution of the attitude of the object with three leaders in a group of floating agents. $f_{\text {int }}=0$ in the first row, $f_{\text {int }}>0$ in the second row, and $f_{\text {int }}<0$ in the third row. From the left to the right, $\tau_{\boldsymbol{e}, \boldsymbol{x}}, \boldsymbol{\tau}_{\boldsymbol{e}, \boldsymbol{y}}$, and $\tau_{\boldsymbol{e}, \boldsymbol{z}}$ are applied, and only the rotation angle about the corresponding axis is displayed. The legends contains the value of $m_{\text {ext }}$ expressed in $\mathrm{Nm}$.

Three leader robots Results of the simulations for a floating system are collected in Fig. 26. The presence of three evenly distributed leader robots in a floating system is sufficient to robustly control the attitude also when $f_{\text {int }}=0$ or $f_{\text {int }}<0$.

A.4 Robustness analysis under noisy measurements

Figures 27 and 28 and 29 contain the values of the three Euler angles roll, pitch and yaw, parameterizing the attitude of the load for one, two and three spread leaders in a group of floating agents, respectively, considering, for each case, $f_{\text {int }}=0$, $f_{\text {int }}>0, f_{\text {int }}<0$. From the figures, one can see that the results are qualitatively analogous to the corresponding ones in the previous section.

\section{References}

A Moosavian SA, Rastegari R, Papadopoulos E (2005) Multiple impedance control for space free-flying robots. Journal of guidance, control, and dynamics 28(5):939-947 

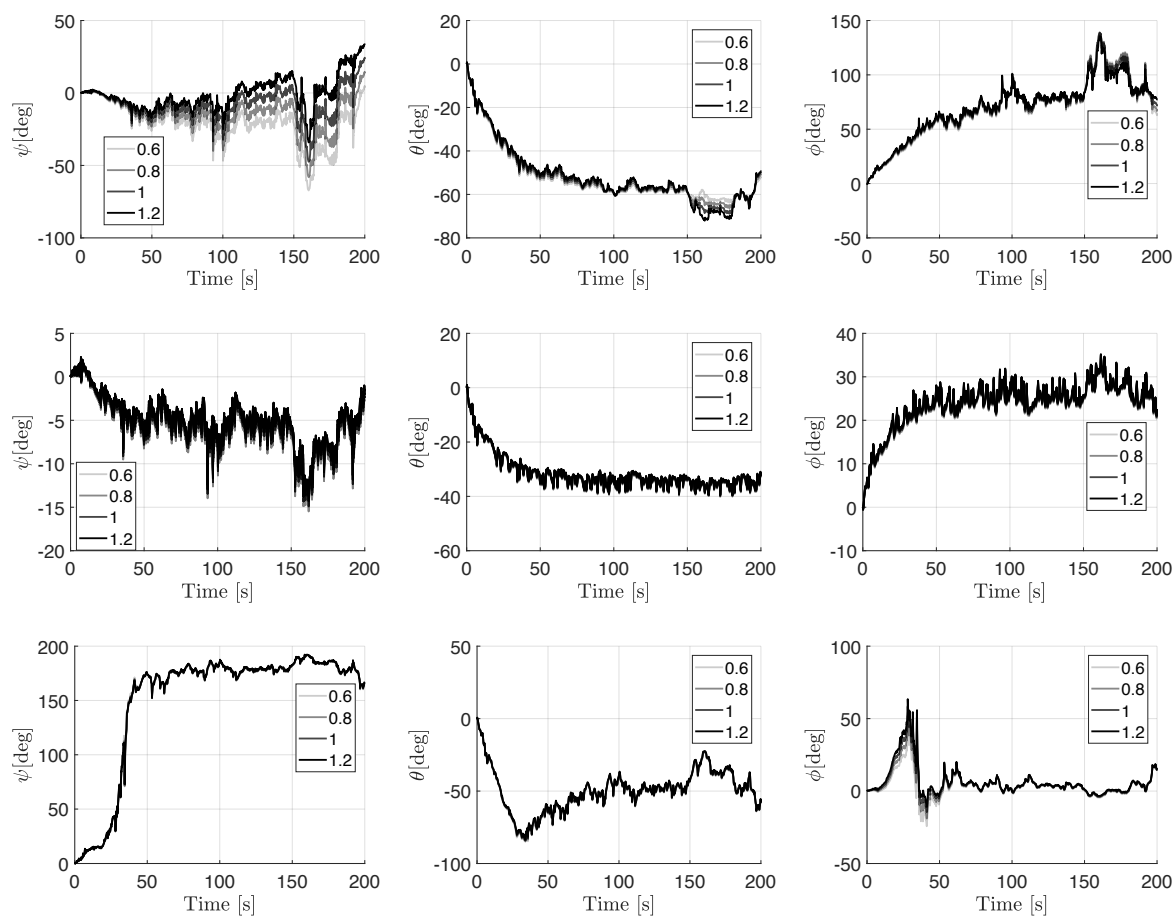

Fig. 27: Evolution of the attitude of the object with one leader in a group of floating agents. The system is subject to an external Gaussian-distributed disturbance torque applied to the object center of mass, with increasing mean value and $0.1 \mathrm{Nm}$ standard deviation. The mean value of the disturbance along the three orthogonal directions is reported in the legend in $\mathrm{Nm}$. $f_{\text {int }}=0$ in the first row, $f_{\text {int }}>0$ in the second, and $f_{\text {int }}<0$ in the third one.

Abbasnejad G, Carricato M (2015) Direct geometrico-static problem of underconstrained cable-driven parallel robots with $\mathrm{n}$ cables. IEEE Trans on Robotics 31(2):468-478

Anitescu M, Potra FA (1997) Formulating dynamic multi-rigid-body contact problems with friction as solvable linear complementarity problems. Nonlinear Dynamics 14(3):231-247

Berman S, Lindsey Q, Sakar MS, Kumar V, Pratt SC (2011) Experimental study and modeling of group retrieval in ants as an approach to collective transport in swarm robotic systems. Proceedings of the IEEE 99(9):1470-1481

Caccavale F, Giglio G, Muscio G, Pierri F (2015) Cooperative impedance control for multiple uavs with a robotic arm. In: 2015 IEEE/RSJ International Conference on Intelligent Robots and Systems (IROS), IEEE, pp 2366-2371

Conti R, Meli E, Ridolfi A, Allotta B (2015) An innovative decentralized strategy for i-auvs cooperative manipulation tasks. Robotics and autonomous systems $72: 261-276$

Di Leonardo R, Angelani L, DellArciprete D, Ruocco G, Iebba V, Schippa S, Conte M, Mecarini F, De Angelis F, Di Fabrizio E (2010) Bacterial ratchet motors. Proceedings of the National Academy of Sciences 

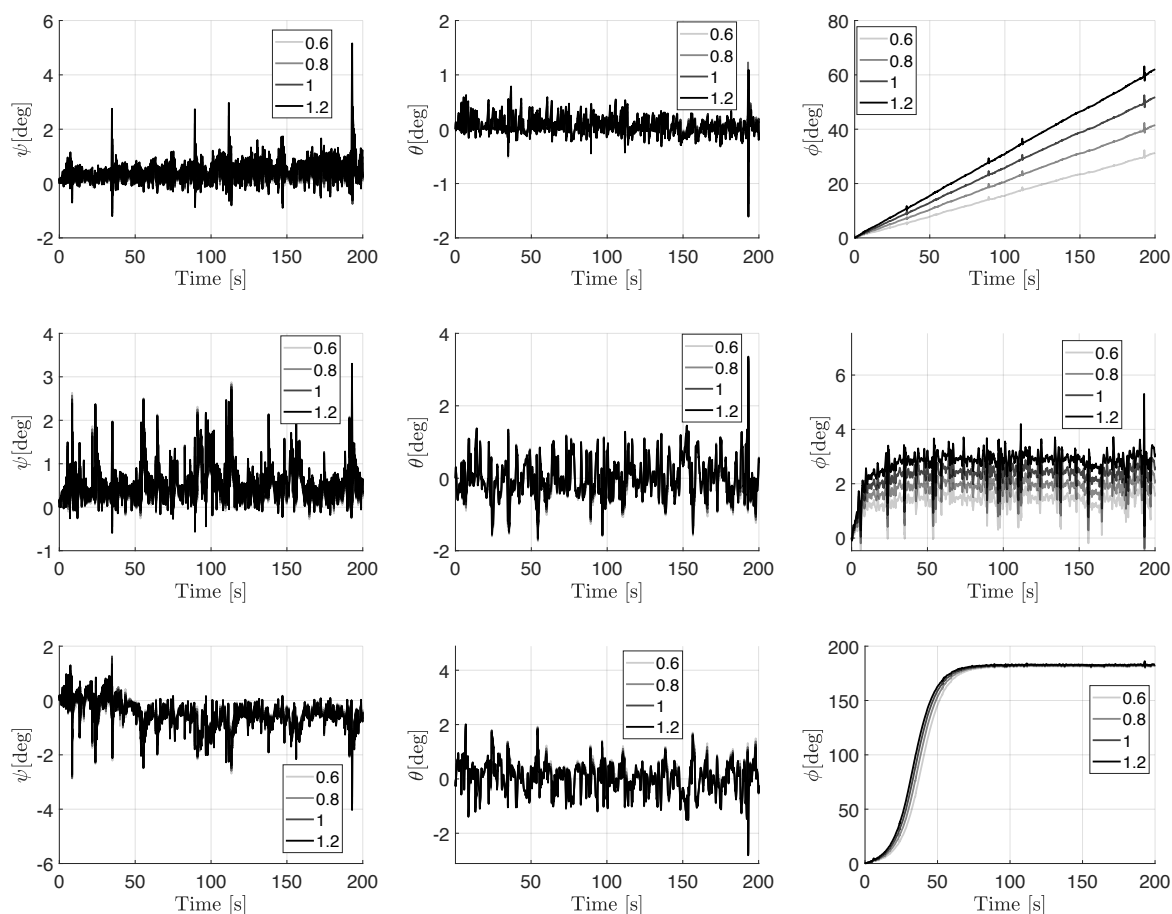

Fig. 28: Evolution of the attitude of the object with two leaders in a group of floating agents. The system is subject to an external Gaussian-distributed disturbance torque applied to the object center of mass, with increasing mean value and $0.1 \mathrm{Nm}$ standard deviation. The mean value of the disturbance along the three orthogonal directions is reported in the legend in $\mathrm{Nm}$. $f_{\text {int }}=0$ in the first row, $f_{\text {int }}>0$ in the second, and $f_{\text {int }}<0$ in the third one.

Farivarnejad H, Berman S (2018) Stability and convergence analysis of a decentralized proportional-integral control strategy for collective transport. In: 2018 Annual American Control Conference (ACC), IEEE, pp 2794-2801

Ferrante E, Turgut AE, Mathews N, Birattari M, Dorigo M (2010) Flocking in stationary and non-stationary environments: a novel communication strategy for heading alignment. In: International Conference on Parallel Problem Solving from Nature, Springer, pp 331-340

Ferrante E, Turgut AE, Huepe C, Stranieri A, Pinciroli C, Dorigo M (2012) Selforganized flocking with a mobile robot swarm: a novel motion control method. Adaptive Behavior 20(6):460-477

Franchi A, Petitti A, Rizzo A (2019) Distributed estimation of state and parameters in multi-agent cooperative load manipulation. IEEE Trans on Control of Network Systems 6(2):690-701, DOI 10.1109/TCNS.2018.2873153

Franks NR (1986) Teams in social insects: group retrieval of prey by army ants (eciton burchelli, hymenoptera: Formicidae). Behavioral Ecology and Sociobiology 18(6):425-429

Franks NR, Sendova-Franks AB, Anderson C (2001) Division of labour within teams of new world and old world army ants. Animal Behaviour 62(4):635-642 

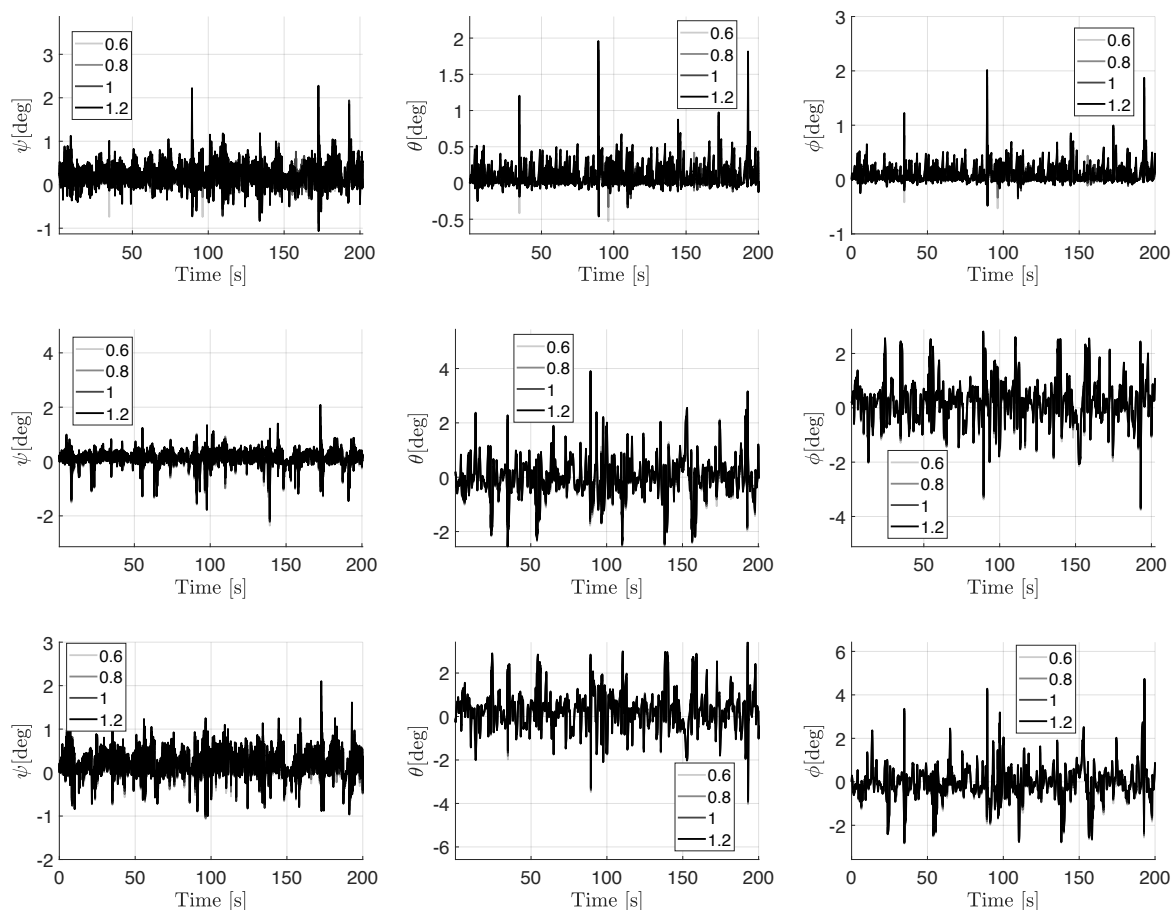

Fig. 29: Evolution of the attitude of the object with three leaders in a group of floating agents. The system is subject to an external Gaussian-distributed disturbance torque applied to the object center of mass, with increasing mean value and $0.1 \mathrm{Nm}$ standard deviation. The mean value of the disturbance along the three orthogonal directions is reported in the legend in $\mathrm{Nm}$. $f_{\text {int }}=0$ in the first row, $f_{\text {int }}>0$ in the second, and $f_{\text {int }}<0$ in the third one.

Fumagalli M, Naldi R, Macchelli A, Forte F, Keemink AQ, Stramigioli S, Carloni R, Marconi L (2014) Physical interaction with the environment. IEEE robotics \& automation magazine 1070(9932/14)

Gabellieri C, Tognon M, Palottino L, Franchi A (2018) A study on force-based collaboration in flying swarms. In: 11th Int. Conf. on Swarm Intelligence, Rome, Italy, pp 3-15

Gassner M, Cieslewski T, Scaramuzza D (2017) Dynamic collaboration without communication: Vision-based cable-suspended load transport with two quadrotors. In: 2017 IEEE Int. Conf. on Robotics and Automation, Singapore, pp $5196-5202$

Grassé PP (1959) La reconstruction du nid et les coordinations interindividuelles chezbellicositermes natalensis etcubitermes sp. la théorie de la stigmergie: Essai d'interprétation du comportement des termites constructeurs. Insectes sociaux $6(1): 41-80$

Ikeura R, Monden H, Inooka H (1994) Cooperative motion control of a robot and a human. In: Robot and Human Communication, 1994. RO-MAN'94 Nagoya, Proceedings., 3rd IEEE International Workshop on, IEEE, pp 112-117 
Kelly I, Keating D (1996) Flocking by the fusion of sonar and active infrared sensors on physical autonomous mobile robots. In: Proceedings of The Third Int. Conf. on Mechatronics and Machine Vision in Practice, vol 1, pp 1-4

Khalil HK (2001) Nonlinear Systems, 3rd edn. Prentice Hall

Kube CR, Bonabeau E (2000) Cooperative transport by ants and robots. Robotics and autonomous systems 30(1-2):85-101

Machado M, Moreira P, Flores P, Lankarani HM (2012) Compliant contact force models in multibody dynamics: Evolution of the hertz contact theory. Mechanism and Machine Theory 53:99 - 121

Manubens M, Devaurs D, Ros L, Cortés J (2013) Motion planning for 6-D manipulation with aerial towed-cable systems. In: 2013 Robotics: Science and Systems, Berlin, Germany

Marino A (2018) Distributed adaptive control of networked cooperative mobile manipulators. IEEE Transactions on Control Systems Technology 26(5):16461660

Mellinger D, Shomin M, Michael N, Kumar V (2013) Cooperative grasping and transport using multiple quadrotors. In: Distributed autonomous robotic systems, Springer, pp 545-558

Nguyen HN, Park S, Lee DJ (2015) Aerial tool operation system using quadrotors as rotating thrust generators. In: 2015 IEEE/RSJ Int. Conf. on Intelligent Robots and Systems, Hamburg, Germany, pp 1285-1291

Pereira PO, Herzog M, Dimarogonas DV (2016) Slung load transportation with a single aerial vehicle and disturbance removal. In: 2016 24th Mediterranean Conference on Control and Automation (MED), IEEE, pp 671-676

Petitti A, Franchi A, Di Paola D, Rizzo A (2016) Decentralized motion control for cooperative manipulation with a team of networked mobile manipulators. In: 2016 IEEE Int. Conf. on Robotics and Automation, Stockholm, Sweden, pp 441-446

Prattichizzo D, Trinkle JC (2008) Grasping. In: Springer handbook of robotics, Springer, pp 671-700

Rubenstein M, Cabrera A, Werfel J, Habibi G, McLurkin J, Nagpal R (2013) Collective transport of complex objects by simple robots: theory and experiments. In: 2013 international conference on Autonomous agents and multi-agent systems, pp 47-54

Schneider SA, Cannon RH (1992) Object impedance control for cooperative manipulation: Theory and experimental results. IEEE Transactions on Robotics and Automation 8(3):383-394

Sieber D, Hirche S (2018) Human-guided multirobot cooperative manipulation. IEEE Transactions on Control Systems Technology (99):1-18

Somvanshi PR, Patel AK, Bhartiya S, Venkatesh K (2015) Implementation of integral feedback control in biological systems. Wiley Interdisciplinary Reviews: Systems Biology and Medicine 7(5):301-316

Sreenath K, Kumar V (2013) Dynamics, control and planning for cooperative manipulation of payloads suspended by cables from multiple quadrotor robots. In: Robotics: Science and Systems, Berlin, Germany

Tagliabue A, Kamel M, Verling S, Siegwart R, Nieto J (2016) Collaborative transportation using MAVs via passive force control. In: 2017 IEEE Int. Conf. on Robotics and Automation, Singapore, pp 5766-5773 
Takubo T, Arai H, Tanie K (2001) Human-robot cooperative handling using virtual nonholonomic constraint in 3-d space. In: Robotics and Automation, 2001. Proceedings 2001 ICRA. IEEE International Conference on, IEEE, vol 3, pp 2680-2685

Tognon M, Franchi A (2017) Dynamics, control, and estimation for aerial robots tethered by cables or bars. IEEE Trans on Robotics 33(4):834-845

Tognon M, Gabellieri C, Pallottino L, Franchi A (2018) Aerial co-manipulation with cables: The role of internal force for equilibria, stability, and passivity. IEEE Robotics and Automation Letters, Special Issue on Aerial Manipulation 3(3):2577 - 2583, DOI 10.1109/LRA.2018.2803811

Tognon M, Tello Chávez HA, Gasparin E, Sablé Q, Bicego D, Mallet A, Lany M, Santi G, Revaz B, Cortés J, Franchi A (2019) A truly redundant aerial manipulator system with application to push-and-slide inspection in industrial plants. IEEE Robotics and Automation Letters 4(2):1846-1851, DOI 10.1109/ LRA.2019.2895880

Tsiamis A, Verginis CK, Bechlioulis CP, Kyriakopoulos KJ (2015) Cooperative manipulation exploiting only implicit communication. In: 2015 IEEE/RSJ International Conference on Intelligent Robots and Systems (IROS), IEEE, pp 864-869

Tuci E, Groß R, Trianni V, Mondada F, Bonani M, Dorigo M (2006) Cooperation through self-assembly in multi-robot systems. ACM Transactions on Autonomous and Adaptive Systems (TAAS) 1(2):115-150

Verginis CK, Nikou A, Dimarogonas DV (2018) Communication-based decentralized cooperative object transportation using nonlinear model predictive control. In: 2018 European Control Conference (ECC), IEEE, pp 733-738

Wang Z, Schwager M (2016) Force-amplifying n-robot transport system (forceants) for cooperative planar manipulation without communication. The International Journal of Robotics Research 35(13):1564-1586

de Wit CC, Siciliano B, Bastin G (1996) Motion and force control. In: Theory of robot control, Springer, chap 4, pp 141-175

Yan Z, Jouandeau N, Cherif AA (2013) A survey and analysis of multi-robot coordination. International Journal of Advanced Robotic Systems 10(12):399 